\title{
GLAUCIO UMAKOSHI
}

Equivalência de instrumentos de qualidade de vida em saúde bucal com a Classificação Internacional de Funcionalidade, Incapacidade e Saúde

São Paulo

2014 


\section{GLAUCIO UMAKOSHI}

Equivalência de instrumentos de qualidade de vida em saúde bucal com a Classificação Internacional de Funcionalidade, Incapacidade e Saúde

\section{Versão Corrigida}

Dissertação apresentada à Faculdade de Odontologia da Universidade de São Paulo, para obter o título de Mestre, pelo Programa de Pós-Graduação em Ciências Odontológicas.

Área de Concentração: Odontologia Social Orientadora: Profa. Dra. Maria Gabriela Haye Biazevic

São Paulo

2014 
Autorizo a reprodução e divulgação total ou parcial deste trabalho, por qualquer meio convencional ou eletrônico, para fins de estudo e pesquisa, desde que citada a fonte.

Catalogação-na-Publicação

Serviço de Documentação Odontológica

Faculdade de Odontologia da Universidade de São Paulo

Umakoshi, Glaucio.

Equivalência de instrumentos de qualidade de vida em saúde bucal com a Classificação Internacional de Funcionalidade, Incapacidade e Saúde / Glaucio Umakoshi ; orientador Maria Gabriela Haye Biazevic. -- São Paulo, 2014.

61p. : fig., tab., graf.; $30 \mathrm{~cm}$.

Dissertação (Mestrado) -- Programa de Pós-Graduação em Ciências Odontológicas. Área de Concentração: Odontologia Social. -- Faculdade de Odontologia da Universidade de São Paulo.

Versão corrigida

1. Qualidade de vida. 2. Neoplasias bucais. 3. Odontalgia. I. Biazevic, Maria Gabriela Haye. II. Título. 
Umakoshi G. Equivalência de instrumentos de qualidade de vida em saúde bucal com a Classificação Internacional de Funcionalidade, Incapacidade e Saúde. Dissertação apresentada à Faculdade de Odontologia da Universidade de São Paulo para obtenção do título de Mestre em Ciências Odontológicas.

Aprovado em: / /2014

\section{Banca Examinadora}

$\operatorname{Prof}(\mathrm{a}) . \operatorname{Dr}(\mathrm{a})$.

Instituição: Julgamento:

$\operatorname{Prof}(a)$. Dr(a).

Instituição: Julgamento:

Prof(a). Dr(a).

Instituição: Julgamento: 
Dedico este trabalho a: meus pais, Cristiane, Alice e Leonardo, com amor e carinho. 


\section{AGRADECIMENTOS}

À Profa. Dra. Maria Gabriela Haye Biazevic, pela paciência, carinho e atenção que teve comigo durante a elaboração deste trabalho e pela amizade e convivência, que - tenho certeza - permanecerá após esta data. Muito Obrigado.

Ao Prof. Dr. Waldyr Antônio Jorge, pelas orientações, cuidado, amizade, fraternidade e por acreditar e depositar sua confiança. Muito Obrigado.

Ao Prof. Michel Edgar Crosato, pela orientação e oportunidade.

Aos Professores da Disciplina de Odontologia Social da Faculdade de Odontologia da Universidade de São Paulo, pela amizade e convivência científica compartilhada.

À Liga de Neoplasias Bucais da Faculdade de Odontologia da Universidade de São Paulo, que disponibilizou seus pacientes para esta pesquisa.

À Clínica de Urgência da Faculdade de Odontologia da Universidade de São Paulo, que disponibilizou seus pacientes para este trabalho.

A todos os pacientes da pesquisa que colaboraram gentilmente para a elaboração deste trabalho.

Às secretárias do Departamento de Odontologia Social da Faculdade de Odontologia da Universidade de São Paulo e a todos os funcionários pelo auxílio durante o trabalho e pela amizade. 


\section{RESUMO}

Umakoshi G. Equivalência de instrumentos de qualidade de vida em saúde bucal com a Classificação Internacional de Funcionalidade, Incapacidade e Saúde [dissertação]. São Paulo: Universidade de São Paulo, Faculdade de Odontologia; 2014. Versão Corrigida.

A Organização Mundial de Saúde (OMS) definiu a qualidade de vida como a percepção do indivíduo sobre si, no contexto de valores em que vive e em relação a: metas, expectativas, parâmetros e relações sociais. O objetivo do estudo foi realizar a equivalência dos instrumentos de qualidade de vida (University of Washington Quality of life (UW-QOL), Brief Core Set Questionnaire - Head and Neck - BCSQ H\&N), em pacientes com câncer de boca, diagnosticados na Liga de Neoplasia Bucal (LINB) e o Oral Health Impact Profile (OHIP-14) para pacientes que buscaram por atendimento de urgência, com a Classificação Internacional de Funcionalidade, Incapacidade e Saúde (CIF). Foram entrevistados 50 pacientes atendidos pela Liga de Neoplasias da FOUSP e 100 pacientes do Setor de Urgências da Clínica Odontológica da FOUSP. Os escores de cada instrumento foram pontuados e, em seguida, verificou-se a frequência de ocorrência dos domínios. As categorias mais frequentes nos instrumentos de qualidade de vida UW-QOL, foram: b152 (Ansiedade - Funções Emocionais), b510 (Funções de ingestão - saliva), b250 (Função gustativa). No questionário BCSQ H\&N, as categorias mais frequentes foram: b510 (Funções de ingestão), b310 (Funções da voz) e b152 (Funções emocionais). No questionário OHIP-14, as categorias mais pontuadas foram: b280 (Sensação de dor), b152 (Funções emocionais) e b130 (Funções da energia e dos impulsos Atividade). Foi observada equivalência entre os domínios dos instrumentos de qualidade de vida e a CIF. Assim, a CIF constitui instrumento útil para avaliar a real situação de saúde do paciente, além dos aspectos clínicos.

Palavras-chave: Câncer Bucal. CIF. Odontalgia. Qualidade de vida. 


\begin{abstract}
Umakoshi G. Equivalence of instruments of quality of life in oral health with the International Classification of Functioning, Disability and Health [dissertation]. São Paulo: Universidade de São Paulo, Faculdade de Odontologia; 2014. Versão Corrigida.
\end{abstract}

The World Health Organization (WHO) defined quality of life as the perception of the individual in his life, in the context of values in which they live and in relation to their goals, expectations, parameters and social relations. The objective of the study was to perform the equivalence of quality of life instruments, University of Washington Quality of Life (UW-QOL), Brief Core Set Questionnaire - Head and Neck (BCSQ $\mathrm{H} \& N$ ) in patients with diagnosed oral cancer in the League of Oral Neoplasms (LINB); and the Oral Health Impact Profile (OHIP-14) for patients who sought for emergency care, with the International Classification of Functioning, Disability and Health (ICF). Fifty patients from the Oral Neoplasms League and 100 patients from the Urgency Clinic at FOUSP were interviewed. Each instrument's scores were registered and the occurrence frequency of each domain was verified. The most frequent categories of instruments in quality of life UW-QOL were: b152 (Anxiety - Emotional functions), b510 (Ingestion functions - saliva), b250 (Taste function). At BCSQ H\&N the most frequent categories were: b510 (Ingestion functions), b310 (Voice functions) and b152 (Emotional functions). In the OHIP-14 questionnaire, the highest scoring categories were: b280 (Sensation of pain), b152 (Emotional functions) and b130 (Energy and drive functions - Activity). Equivalences was observed among the instruments' domains and ICF. Thus, ICF is a useful tool to perform real evaluations on the patients' health, adding information to the clinical aspects.

Keywords: Oral Cancer. ICF. Toothache.Quality of Life. 


\section{LISTA DE TABELAS}

Tabela 2.1 - Categorias da CIF identificadas no BCSQ H\&N

Tabela 5.1 - Informações sócio-demográficas e localização anatômica dos tumores dos pacientes atendidos na Liga de Neoplasias Bucais (LINB) da FOUSP 30

Tabela 5.2 - Média da pontuação geral e segundo domínios específicos do University of Washington Quality of Life (UW-QoL) dos pacientes atendidos na Liga de Neoplasias Bucais (LINB) da FOUSP.

Tabela 5.3 - Média da pontuação geral e segundo domínios específicos da Seção 1 do Brief Core Set Questionnaire Head and Neck (BCSQ-H\&N) dos pacientes atendidos na Liga de Neoplasias Bucais (LINB) da FOUSP

Tabela 5.4 - Média da pontuação geral e segundo domínios específicos da Seção 2 do Brief Core Set Questionnaire Head and Neck (BCSQ-H\&N) dos pacientes atendidos na Liga de Neoplasias Bucais (LINB) da FOUSP

Tabela 5.5 - Média da pontuação geral e segundo domínios específicos da Seção 3 do Brief Core Set Questionnaire Head and Neck (BCSQ-H\&N) dos pacientes atendidos na Liga de Neoplasias Bucais (LINB) da FOUSP

Tabela 5.6 - Informações sócio-demográficas e motivo da consulta dos pacientes atendidos pelo setor de Urgências da FOUSP

Tabela 5.7 - Média da pontuação geral e segundo domínios específicos do Oral Health Impact Profile (OHIP) dos pacientes atendidos pelo Setor de Urgências da FOUSP

Tabela 5.8 - Categorias da CIF identificadas nas questões do questionário UW-QOL .35

Tabela 5.9 - Categorias da CIF identificadas no questionário OHIP-14 36

Tabela 5.10-Categorias mais presentes da CIF, segundo frequência de aparecimento nas respostas dos questionários OHIP-14, UQ-QOL e BCSQ H\&N 


\section{LISTA DE ABREVIATURAS E SIGLAS}

$\begin{array}{ll}\text { BCSQ H\&N } & \text { Brief Core Set Questionnaire Head and Neck } \\ \text { CCP } & \text { Câncer de Cabeça e Pescoço } \\ \text { CID - 10 } & \text { Classificação Internacional de Doenças - 10a Versão } \\ \text { CIF } & \text { Classificação Internacional de Funcionalidade, } \\ & \text { Incapacidade e Saúde } \\ \text { CNS - MS } & \text { Conselho Nacional de Saúde - Ministério da Saúde } \\ \text { EORTC } & \text { European Organization for Research and Treatment of } \\ \text { FOUSP } & \text { Cancer } \\ \text { HNC } & \text { Faculdade de Odontologia da Universidade de São Paulo } \\ \text { ICF } & \text { Head and Neck Cancer } \\ \text { ICIDH } & \text { International Classification Functioning, Disability and } \\ \text { INCA } & \text { Health } \\ \text { LINB } & \text { International Classification of Impairments, Disabilities and } \\ \text { MOS SF - 36 } & \text { Handicaps } \\ \text { OHIP } & \text { Instituto Nacional de Câncer } \\ \text { OMS } & \text { Liga de Neoplasias Bucais } \\ \text { QV } & \text { Medical Outcomes Short form - 36 } \\ \text { QVRS } & \text { Oral Health Impact Profile } \\ \text { RI } & \text { Organização Mundial da Saúde } \\ \text { SIP } & \text { Qualidade de Vida } \\ \text { SUS } & \text { Qualidade de Vida Relacionada a Saúde } \\ \text { UW - QOL } & \text { Rehabilitation International } \\ \text { WHO } & \text { Sickness impact Profile } \\ \text { WHOQOL } & \text { Sistema Único de Saúde } \\ \text { Universidade de Washington } \\ \text { World Health Organization } \\ \text { World Health Organization Quality of Life } \\ \end{array}$




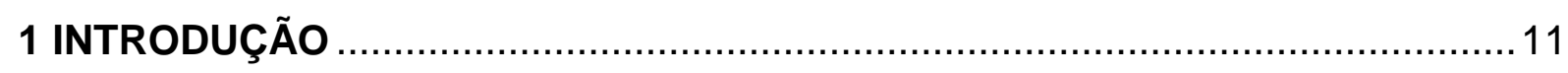

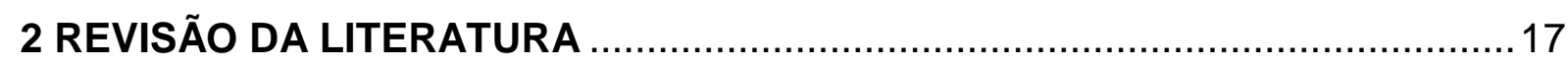

2.1 Classificação Internacional de Funcionalidade Incapacidade e Saúde ................17

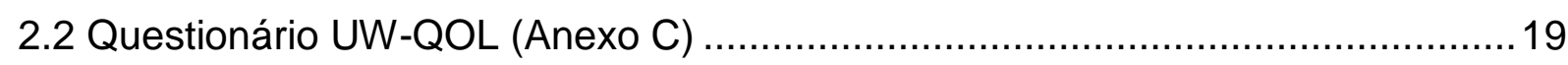

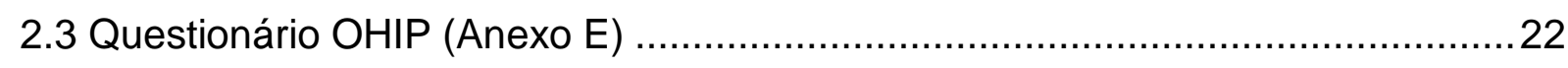

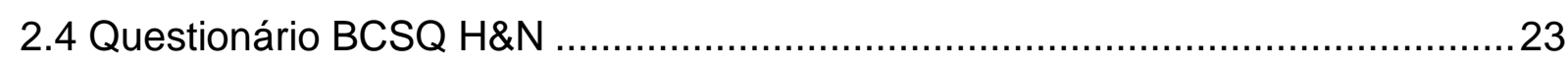

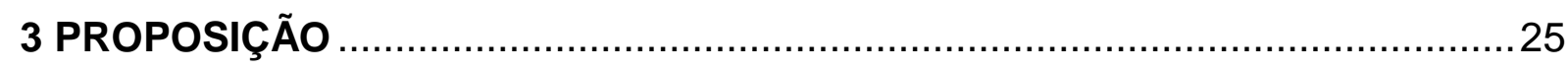

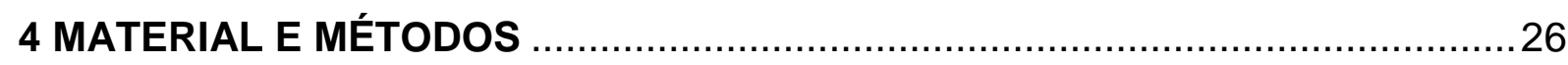

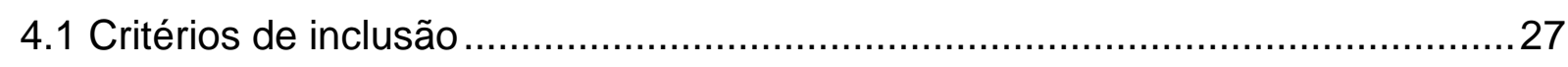

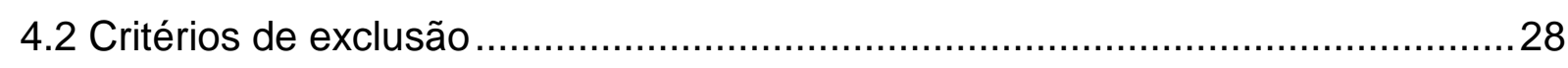

5 RESULTADOS

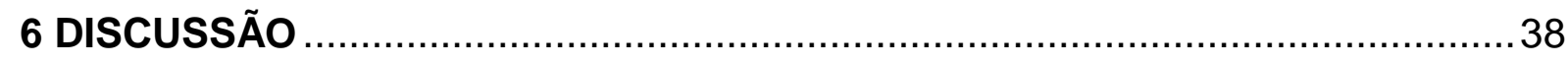

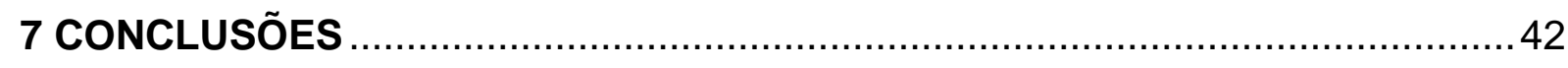

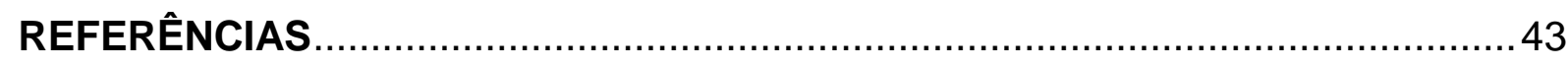

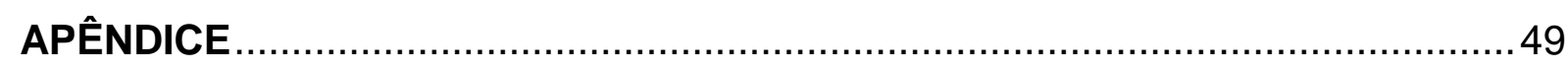

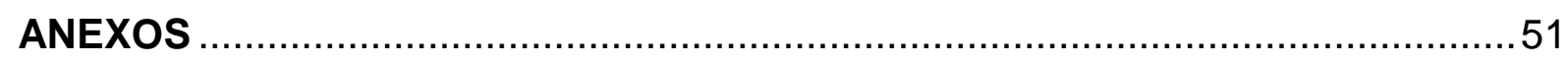




\section{INTRODUÇÃO}

A qualidade de vida (QV) como definição pode ser empregada em duas propostas: (1) na linguagem usual e cotidiana, por pessoas da população em geral; e (2) na pesquisa científica, em diferentes áreas de conhecimentos (economia, sociologia, educação, medicina, enfermagem, psicologia) e demais especialidades da saúde (Bowling; Brazier, 1995; Rogerson, 1995).

O termo "Qualidade de vida" tem sido utilizado para avaliar o estado de saúde e o impacto das aplicações terapêuticas em pacientes com diferentes doenças. A Organização Europeia para Pesquisa e Tratamento de Câncer (EORTC), criada em 1980, tem coordenado estudos em várias áreas da Oncologia, inclusive sobre qualidade de vida, nos países da Europa, América e Oceania. Reiterou a complexidade e dificuldade que envolve o conceito de QV, bem como a necessidade de novas investigações multicêntricas e transculturais (Hammerlid et al., 2001).

Um grupo de trabalho da Organização Mundial de Saúde, a fim de obter definição sintética, definiu qualidade de vida como:

[...] a percepção do indivíduo de sua posição na vida, no contexto e sistema de valores em que vive, e em relação a suas metas, expectativas, parâmetros e relações sociais. É um conceito de larga abrangência, afetando de modo complexo a saúde física e da pessoa, seu estado psicológico, nível de independência, relacionamento social e suas relações com características salientes do ambiente. (The WHOQOL Group, 1994, p. 49).

Diversos trabalhos clínicos em saúde têm como objetivo a busca pela melhoria da qualidade de vida dos pacientes. Nessas pesquisas, têm sido discutidos métodos para tratamento e prevenção de doenças, motivo pelo qual surge a necessidade de padronizar a avaliação e melhorar a definição. A ciência médica definiu o conceito, o entendimento por qualidade de vida (Nobre, 1995), definição esta que deveria aplicar-se a todas as pessoas, independentemente do seu estado de saúde. De modo que não poderia ser determinada por condições ambientais ou por comportamento influenciado pelo meio social em que se vive.

Assim, Qualidade de Vida é definida como algo inerente ao indivíduo, às características pessoais, tanto nos aspectos biológicos, como nos hábitos adquiridos 
durante a vida. Isto é, informações que somente o indivíduo pode avaliar e repassar ao pesquisador, livre de influências externas a ele. Portanto, a qualidade de vida poderia ser definida como sensação de conforto, bem estar ou realização plena no desempenho de funções psíquicas, sociais e físicas dentro da própria realidade com familiares, trabalho e valores da comunidade em que vive (Miettinem, 1987).

Atualmente, a associação de qualidade de vida e aspectos gerais de saúde (que inclui a saúde bucal) tem sido discutida amplamente. Embora a maioria das doenças orais não seja fatal, levam a significativa morbidade e geram consequências sociais e psicológicas que afetam a qualidade de vida dos pacientes (Locker; Gibson, 2005; Cohen-Carneiro et al., 2011). Tal associação tem fundamental importância na percepção das pessoas, demarcando uma questão subjetiva à própria imagem, à necessidade e à busca por cuidados odontológicos (Walter et al., 2007). A literatura registra, inclusive, a existência de diferentes necessidades de tratamento expressa pelo cirurgião-dentista e aquela referida pelo paciente (Lundegren et al., 2004). E, ainda, existem evidências de que a autopercepção negativa da saúde mostra relação com indicadores de iniquidades sociais, exacerbando a ação deletéria dos determinantes sociais de saúde (Cremonese et al., 2010; Sheiham et al., 2011).

$\mathrm{Na}$ ciência especializada, ao compreender essa questão, surge a tentativa de criar e validar instrumentos que avaliem o impacto da saúde bucal na qualidade de vida dos indivíduos, denominados na literatura como "indicadores sociodentais" (Reisine, 1981). Entre esses indicadores, destaca-se pelo seu amplo uso o Perfil de Impacto da Saúde Bucal ou Oral Health Impact Profile, mais conhecido como OHIP (Slade; Spencer, 1994).

Em particular, nos pacientes com câncer de cabeça e pescoço (CCP), na autoavaliação sobre a qualidade de vida esse indicador é útil, pois auxilia nas medidas de avaliação das efetividades dos tratamentos. Apesar dos avanços de diagnóstico e tratamentos, esses tumores estão associados a: desfiguramento e disfunções que afetam domínios vitais (Testa; Simonson, 1996).

O câncer bucal foi estimado pelo Instituto Nacional do Câncer José Alencar Gomes da Silva (INCA) que, em 2014, recebeu 11.280 casos novos de câncer da 
cavidade oral em homens e 4.010 em mulheres. Esses valores correspondem a um risco de 11,54 novos casos a cada 100 mil homens, e 3,92 a cada 100 mil mulheres. Sem considerar tumores de pele, o câncer da cavidade oral em homens é o quarto mais frequente na região sudeste $(15,48 / 100$ mil) (INCA, 2014).

A Classificação Internacional de Doenças (CID) surgiu, em princípio, para catalogar universalmente as causas de morte. Passou a ser utilizada e, com o crescente interesse, foi ampliada para codificar estados de saúde de pacientes hospitalizados, consultas ambulatoriais e atenção primária, além de ser utilizada também sobre morbidade. A Décima Revisão, "Classificação Estatística Internacional de Doenças e Problemas Relacionados à Saúde", ou de forma abreviada "CID-10", é a revisão mais recente da "Classificação de Bertillon" de 1893 (Organização Mundial de Saúde, 1996), que era uma classificação de causas de morte. Somente a partir da Sexta Revisão passou a ser a classificação que utilizou todas as doenças e os motivos de consultas, de modo a possibilitar seu uso em morbidade (Laurenti, 1991).

Da análise dos comentários de muitos usuários da classificação, verificou-se a necessidade de serem incluídas não somente informações diagnósticas, mas também outras que complementassem questões relacionadas à saúde. Baseado nesse conceito e com foco na CID, procurou-se atender às necessidades de informações diagnósticas em conjunto com outras classificações. Em consequência, na Décima Revisão, foi aprovado o desenvolvimento de uma "família" de classificações para as diversas áreas de saúde (Laurenti, 1991; Organização Mundial da Saúde, 1996; Üstun, 2002).

Somente a codificação de diagnóstico não permitiria analisar, em toda a amplitude, as consequências do diagnóstico no dia a dia dos pacientes. Por isso a OMS, a fim de fornecer estrutura para estudo de doenças e, pela necessidade de cobrir questões não alcançadas pela CID, publicou em 1980 a International Classification of Impairments, Disabilities and Handicaps (Classificação Internacional de Deficiências, Incapacidades e Desvantagens) conhecidas pelas respectivas siglas: ICIDH ou CIDID, em caráter de teste, cujas categorias correspondem às consequências das doenças (Organização Mundial da Saúde, 1989; Casado, 2001; Üstun, 2002). 
A versão, agora chamada de CIF (Organização Mundial da Saúde, 2003), foi desenvolvida na década de 90 e obteve a aprovação para uso internacional em 2001 (Üstun, 2002; Organização Mundial da Saúde, 2003), produzindo conhecimento e conceitos diversos dos anteriores com relação à saúde (Egea; Sarabia, 2001; World Health Organization, 2001). Várias organizações, entre elas Rehabilitation International (RI), tiveram importante participação nas conceituações ao longo das revisões da ICIDH/CIDID até a versão final da CIF (Casado, 2001).

O uso concomitante - de ambas as condições do estado de saúde, CID e CIF, - traz vantagens, pois fornece panorama mais abrangente da situação de saúde dos indivíduos. Por isso, é importante compreender a relação entre esses dois conceitos que proporcionam abordagem sistemática e padronizada, quando se busca relacionar o estado de saúde medido para a CIF (Cieza et al., 2002).

Com a utilização em larga escala, a CIF permite nova nomenclatura e visa a estabelecer protocolos voltados às pessoas com deficiência ou incapacidade, o que ajuda a estabelecer a igualdade de oportunidades. A participação do poder público para mais divulgação e inserção da CIF é importante para melhor definição e avaliação das incapacidades e deficiências (Di Nubila; Buchalla, 2008).

O Plenário do Conselho Nacional de Saúde do Ministério da Saúde, em sua 233a reunião, estabeleceu que a CIF seja utilizada no Sistema Único de Saúde (SUS), inclusive na saúde suplementar (Resolução № 452 de 10 de maio 2012 CNS - MS), uma vez que o desenvolvimento de políticas públicas em saúde devem destacar funcionalidade e incapacidade (para pessoas com ou sem deficiência) e considerando que o Brasil, membro da OMS, ainda não incorporou a CIF nesse sistema (Brasil, 2012).

A CIF foi introduzida como base para avaliação da funcionalidade, e o conjunto de cores sets em câncer de cabeça e pescoço (CCP) pode oferecer lista abrangente de aspectos relevantes, auxiliar os profissionais de saúde em diferentes técnicas de abordagens ou tratamentos para reabilitação. A participação da equipe de saúde em grande escala, proporciona ativamente o processo de desenvolvimento de uma rede mundial aceita e uma ferramenta padronizada na avaliação da doença (Tschiesner et al., 2009b). 
A avaliação prospectiva de sobrevivência e qualidade de vida pode contribuir para antecipar intervenções destinadas a reduzir a incidência de limitações funcionais em pacientes com câncer de boca e de orofaringe (Biazevic et al., 2008).

Os diversos instrumentos de qualidade de vida, genéricos ou específicos, permitem explorar um efeito de uma doença sobre a vida da pessoa, bem como avaliar aspectos, como desconfortos físicos e emocionais, além de contribuir para a determinação do tratamento (Leal, 2008).

A versão validada do questionário da Universidade de Washington (UW-QOL) é uma valiosa ferramenta para avaliar com precisão a qualidade de vida de pacientes brasileiros com câncer de cabeça e pescoço (Vartanian et al., 2006).

A CIF também foi utilizada para avaliar a funcionalidade e a incapacidade, em 19 itens, num conjunto de questões rápidas em relação ao CCP. Este estudo desenvolveu um questionário de autopreenchimento, a partir dos itens do conjunto de questões rápidas (Brief Core Set Questionnaire - Head and Neck - BCSQ-H\&N), cujas respostas foram posteriormente comparadas às do questionário BCSQ-H\&N com a Universidade de Washington v.4 (UW-QOLv4) e aos resultados BCSQ-H\&N, com avaliação clínica (Rogers et al., 2010).

Ao se desenvolver, no sul da Austrália, o Oral Health Impact Profile (OHIP), instrumento criado para avaliar o impacto social da doença bucal, Slade e Spencer, (1994) notaram que o OHIP foi capaz de detectar associação, previamente observada, entre o impacto social e a necessidade percebida de tratamento. O OHIP é composto pelos seguintes domínios: limitação funcional, dor física, desconforto psicológico, incapacidade física, psicológica e social e deficiência na realização das atividades cotidianas (Miotto et al., 2012).

O OHIP- na sua forma simplificada, chamada de OHIP-14 - é questionário capaz de avaliar a qualidade de vida em relação às condições de saúde bucal (Biazevic et al., 2004). A validação do instrumento mostrou propriedades psicométricas semelhantes às originais (Almeida et al., 2004). 
As diversas medidas de qualidade de vida, relacionadas à saúde, devem permitir a avaliação multidirecional do impacto da doença ou o tratamento do indivíduo e não somente o impacto no aspecto físico (Murphy et al., 2007).

A importância da avaliação da qualidade de vida, por meio da utilização de instrumentos apropriados, pode contribuir para antecipar intervenções voltadas à redução da ansiedade causada pela doença, auxiliando no tratamento e prognóstico do câncer de cabeça e pescoço. 


\section{REVISÃO DA LITERATURA}

A avaliação da QVRS não deve ser utilizada apenas em pesquisa, mas na prática cínica como indicador de respostas a tratamentos e na evolução de cuidados. e por ser uma avaliação embasada em características individuais, crenças e expectativas, a QV deve ser avaliada pelo paciente e não pelo profissional (Sayed et al. 2009). Sendo importante identificar quais instrumentos de qualidade de vida que melhor se ajustam a estes objetivos (Vido; Fernandes, 2007).

2.1 Classificação Internacional de Funcionalidade Incapacidade e Saúde

O enfoque principal da CIF é a influência dos fatores contextuais (ambientais e pessoais) e seus impactos, tanto positivos quanto negativos, nas três dimensões das condições de saúde: estrutura e função do corpo, atividade e participação social (Organização Mundial da Saúde, 2003).

Optou-se por organizar a pesquisa em duas partes:

Parte 1- Funcionalidade e Incapacidade, composta por Funções do corpo/ estruturas do corpo e atividade/participação.

Parte 2- Fatores contextuais, composta por fatores ambientais e fatores pessoais (estes não classificados atualmente na CIF).

Cada componente é identificado por prefixos, em cada código: $\mathbf{b}$ (do inglês body, corpo) para funções do corpo; s (do inglês structure) para estruturas do corpo; d (do inglês domain) para atividades e participação e, e (do inglês environment) para fatores ambientais (Organização Mundial da Saúde, 2003).

Nessa classificação, todos os domínios de saúde e os conteúdos relacionados interagem e apresentam a mesma relevância para descrever o processo de funcionalidade e incapacidade. Essa classificação constitui, portanto, abordagem com múltiplas perspectivas para descrever a funcionalidade e a 
incapacidade como processo interativo e evolutivo (Organização Mundial da Saúde, 2003).

Abaixo estão descritos os componentes da CIF:

1 - "Estrutura do corpo" - definida como as partes anatômicas do corpo como órgãos e membros; e "funções do corpo": fisiológicas e psicológicas do sistema corporal (Organização Mundial da Saúde, 2003).

No contexto da Odontologia, mais especificamente em pacientes diagnosticados com câncer de cabeça e pescoço (tratados ou não), há deficiência em alguma estrutura do sistema estomatognático, o que geralmente tem efeitos na função do indivíduo (paladar, olfato, mastigação, deglutição entre outras).

Figura 4.1 - Interação entre os componentes da CIF

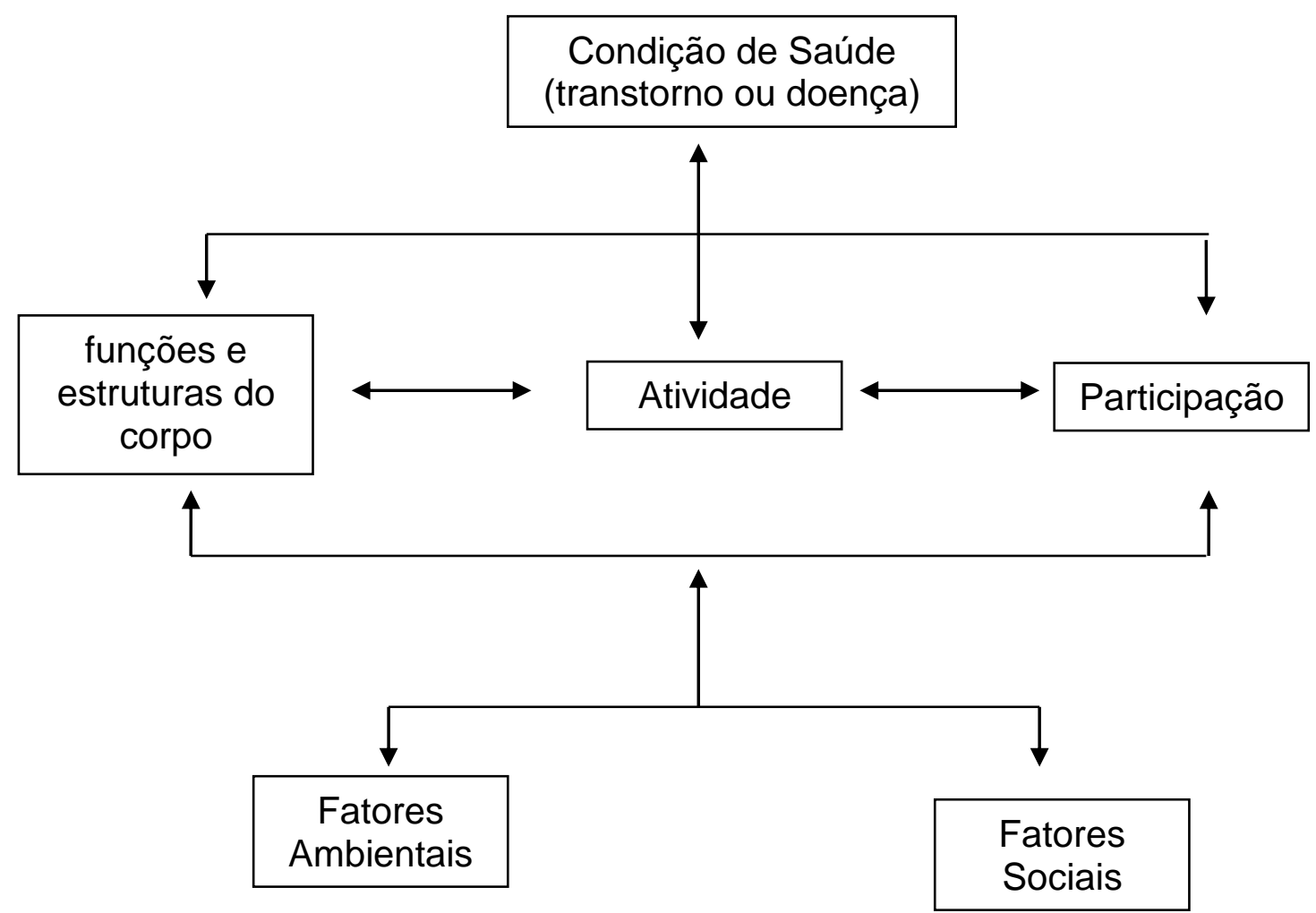

Fonte: Organização Mundial da Saúde (2003). 
Com a aplicação dos questionários, UW-QOL e o anexo BCSQ-H\&N, para os pacientes da LINB da FOUSP; e o OHIP-14, para os pacientes da Clínica de Urgência da FOUSP, foi realizada a equivalência com a CIF, de modo que as perguntas dos questionários foram associadas aos respectivos capítulos e códigos correspondentes.

\subsection{Questionário UW-QOL (Anexo C)}

Através do estudo de Hassan e Weymuller Jr (1993), o questionário de QV da Universidade de Washington foi validado e comparado aos resultados de sua aplicação nos 75 pacientes com câncer de cabeça e pescoço (CCP), desde o estágio pré-operatório e controlado após três meses do início do tratamento, com os dados obtidos através dos outros questionários já validados: o Karnofsky Performance Scale e o Sickness Impact Profile - (SIP). Os questionários foram comparados nos fatores: aceitabilidade, validade e responsividade. O questionário UW-QOL, quanto à aceitabilidade, obteve $97 \%$ de aceitação, sendo preterido aos demais pela facilidade em responder e ser mais conciso. Quanto à validade, o UWQOL obteve bons indicadores de concordância, considerado gold standard; e a responsividade foi superior, em comparação aos outros dois, por detectar mudanças na QV associadas a modificações no estado clínico dos pacientes. O UW-QOL foi desenvolvido especialmente para pacientes com CCP e continha originalmente 9 itens.

Após análise da performance do questionário UW-QOL, foram removidos do questionário dois itens: a secura da boca e outro relativo ao trabalho, por apresentarem pouca relação com a pontuação dos demais itens. Após a aplicação, em mais de 500 pacientes, foi comprovada a facilidade de aplicação, interpretação, aceitabilidade e validade, além de requerer pouco treinamento de examinadores para aplicá-lo (Weymuller Jr et al., 2001).

A comparação, entre os questionários UW-QOL, o Medical Outcomes Short Form 36 (MOS SF-36) e os questionários da EORTC (estes já validados), sobre a aplicação em pacientes que fariam sua primeira cirurgia por apresentarem câncer 
bucal, mostrou certa deficiência, pois os pacientes tiveram dificuldade em responder a questionários extensos. O estudo concluiu que o UW-QOL era apropriado para uso na prática clínica, graças ao preenchimento simples e rápido, bem como ter dados de fácil processamento, além de avaliar o estado funcional dos doentes (Rogers et al., 1998).

Na segunda versão do questionário, publicada em 1997, foi adicionado um estudo com o propósito de avaliar a importância de cada domínio da QV em pacientes com câncer de cabeça e orofaringe, através do UW-QOL e foi considerado o único questionário de QV aplicado a cabeça e pescoço que realiza essa avaliação. A amostra retratou 48 pacientes, com média de 60 anos de idade, entrevistados no estágio pré-operatório, seis meses e um ano após a cirurgia. $\mathrm{O}$ trabalho concluiu que a avaliação de QV dos pacientes considerou críticos os domínios de deglutição, mastigação, fala e dor, o que indica a utilidade de avaliação para planejamentos terapêuticos individualizados (Rogers et al., 2002).

Com o questionário UW-QOL versão 4, foram avaliados os seis primeiros meses após o tratamento de câncer de cabeça e pescoço e $70 \%$ dos casos relacionados a boca e orofaringe. Verificou-se que os escores aumentaram consideravelmente para ansiedade, dor e humor, quando comparados ao início do tratamento, e os domínios de fala e deglutição foram considerados importantes, independentemente do estágio do tratamento (Radford et al., 2004).

Com a aplicação do questionário UW-QOL foram identificadas diferenças entre o grupo de 121 pacientes com diagnóstico de câncer bucal, divididos em três grupos, com escores de 40 a 69 (30 pacientes), 70 a 89 (66 pacientes) e 90 a 100 (25 pacientes). Identificou-se um grupo com tendência de melhora de QV ao longo do tempo, diferenciados dos que teriam de apresentar essa melhora. Relacionou-se tamanho maior de tumor a escores menores; inversamente proporcional aos pacientes com tumores pequenos. Os itens: fala, mastigação e desfiguramento, foram as principais queixas após seis meses de tratamento, condição que persistiu após um ano do início do tratamento (Rogers et al., 2000).

O estado funcional dos 48 pacientes com câncer bucal e de orofaringe, tratados com cirurgia, foi investigado através do UW-QOL. O item dor foi o sintoma 
mais relatado no momento do diagnóstico, seguido por desfiguramento, dificuldades para engolir, falar e, ainda, problemas nos ombros. Os resultados em relação à QV após um ano da cirurgia mostraram dor, problemas com mastigação e desfiguramento como os domínios que apresentaram mais modificação em relação aos dados iniciais (Rogers et al., 1999).

Um estudo teve como objetivo explorar os 11 domínios no exame clínico (abertura de boca, aparência, dieta, estado dental, competência dos lábios, movimento da língua, mucosa oral, fala, sensação da boca, movimento do ombro e incontinência salivar), e a QV dos pacientes com câncer de cabeça e pescoço com o questionário UW-QOL. Verificou-se que o fator de importância clínica para 0 prognóstico de QV estava diretamente relacionado ao tamanho do tumor: quanto menor o tumor, maior a pontuação e melhor a função clínica. Como resultado, comparado ao pré-operatório, os melhores escores acumulados foram obtidos para os pacientes com pequenos tumores e melhores escores funcionais (Rogers et al., 2002).

Vartanian et al. (2004) aplicaram o UW-QOL, versão 3 em Português, em pacientes com câncer de cavidade bucal e orofaringe, e concluíram que os pacientes que relataram maior piora na aparência foram mulheres com menos de 60 anos, com tumores em estágios T3 e T4. Pacientes com linfonodos no pescoço relataram piora nos escores de recreação, mastigação, engolir, fala e ombros. $O$ tratamento (cirurgia com radioterapia) foi associado com piora dos escores de aparência, mastigação, engolir, recreação e fala. Além disso, observaram que a diferença na composição dos escores dos pacientes com comprometimento de linfonodos e tratamento combinado (cirurgia e radioterapia) não foi estatisticamente significante, apesar de ser clinicamente relevante.

Foram adicionados, na quarta versão do UW-QOL, dois domínios específicos para avaliação de ansiedade e disposição, a partir da associação de vivência e tratamento da patologia. O relato dos pacientes com câncer de cabeça e pescoço, em relação ao questionário UW-QOL foi de boa aceitação e responsividade (Hassan; Weymuller Jr, 1993). 


\subsection{Questionário OHIP (Anexo E)}

O Oral Health Impact Profile (OHIP) foi desenvolvido com o objetivo de proporcionar medida abrangente de autorrelato para disfunção, desconforto e incapacidade atribuída às condições de saúde bucal. Esses impactos foram destinados a complementar indicadores epidemiológicos bucais tradicionais de doença clínica, fornecendo assim informações sobre o "peso da doença", nas populações, e a eficácia dos serviços de saúde na redução dessa carga de doença (Tugwell et al., 1985).

O OHIP, em sua versão original, é composto por 49 questões (Slade; Spencer, 1994). Em 1997, uma versão abreviada foi criada, o OHIP-14, com 14 questões (Slade, 1997).

O instrumento contempla sete dimensões do impacto a ser medido: limitação funcional, dor física, desconforto psicológico, incapacidade física, incapacidade psicológica, incapacidade social e deficiência. As respostas são dadas de acordo com uma escala Likert como: 0 = nunca, $1=$ raramente, 2 = às vezes, 3 = frequentemente e 4 = sempre. Quanto mais alto o valor atribuído pelo respondente, pior é a autopercepção do impacto da saúde bucal na qualidade de vida. O OHIP considera as consequências sociais dos problemas bucais de acordo com a percepção dos próprios indivíduos afetados (Slade; Spencer, 1994).

A ampla aplicabilidade do OHIP tem sido demonstrada em distintas culturas e diferentes países, com o intuito de, eventualmente, associar fatores sociais, econômicos, demográficos e comportamentais (lkebe et al., 2004; Walter et al., 2007; Nguyen et al., 2011).

O OHIP-14, em sua forma abreviada, é frequentemente citado por ser mais simples na aplicação, contrariamente à forma original, com 49 questões, que despende muito tempo. Portanto esse formato longo tem sido menos indicado (Slade, 1997). Essa pode ser a justificativa para a utilização majoritária da versão curta nos estudos revisados (Brennan et al., 2006; Nguyen et al., 2011). 
O instrumento OHIP é importante auxiliar no esclarecimento de necessidades em saúde bucal e na elaboração de estratégias para controle/ redução de doenças e promoção da saúde bucal com impacto positivo na qualidade de vida (Gabardo et al., 2013).

A literatura tem registrado, inclusive, a existência de dissonância entre a necessidade de tratamento, expressa pelo cirurgião-dentista, e aquela referida pelo paciente (Lundegren et al., 2004). Além disso, há evidências de que a autopercepção negativa da saúde guarda relação com indicadores de iniquidades sociais, o que evidencia a ação deletéria dos determinantes sociais de saúde (Cremonese et al., 2010; Sheiham et al., 2011). A comunidade científica especializada, ao compreender criticamente essa questão, tem procurado criar e validar instrumentos que avaliem o impacto das condições de saúde bucal na qualidade de vida dos indivíduos.

\subsection{Questionário BCSQ H\&N}

Existem vários questionários específicos para o câncer de cabeça e pescoço, cada um com características próprias. O core set da CIF foi desenvolvido para diversas condições de saúde, incluindo câncer de cabeça e pescoço (Rogers et al., 2010). Aliás, a finalidade do core set da CIF é selecionar um conjunto de doenças específicas que podem servir como padrões mínimos para abrangente avaliação e documentação do funcionamento e saúde dos estudos clínicos, reuniões clínicas e avaliações multiprofissionais.

O conjunto de cores sets da CIF foi criado em dois níveis: um core set breve, para definir categorias de padrões mínimos, como avaliar e reportar o funcionamento e a saúde em qualquer paciente com CCP; e um core set compreensivo, aplicável a avaliação multidisciplinar.

Enquanto um core set compreensivo da CIF para CCP deve incluir a totalidade dos problemas na funcionalidade dos pacientes, o core set breve visa a incluir apenas as categorias mais importantes entre países e profissões de saúde. 
Uma primeira versão do ICF Core Set para HNC foi criada por meio de consenso internacional e multidisciplinar (Rogers et al., 2002; Tschiesner et al., 2008; Tschiesner et al., 2009a; Tschiesner et al., 2009b; Tschiesner et al., 2009c).

Tabela 2.1 - Categorias da CIF identificadas no BCSQ H\&N

\begin{tabular}{ll}
\hline Funções do corpo $(\mathrm{n}=6)$ & Significado da codificação \\
\hline b510 & Funções de ingestão \\
b280 & Função de dor, sensação de dor \\
b310 & Função de voz \\
b152 & Funções emocionais \\
b130 & Energia e função de movimento \\
B440 & Funções de respiração \\
\hline Estruturas do corpo $(\mathrm{n}=4)$ & \\
\hline s320 & Estrutura da boca \\
s330 & Estrutura da faringe \\
s340 & Estrutura da laringe \\
s710 & Estrutura da região da cabeça e pescoço \\
\hline Atividades e participação $(n=3)$ & \\
\hline d550 & Comer \\
d560 & Beber \\
d230 & Realizar rotina diária \\
d330 & Fala \\
d760 & Relações familiares \\
d870 & Auto-suficiência econômica \\
\hline Fatores Ambientais $(n=3)$ & \\
\hline e310 & Família imediata \\
e110 & Produto ou substância para consumo pessoal \\
e355 & Profissionais de saúde \\
\hline Fonte: Rogers et al. $(2010)$ & \\
&
\end{tabular}




\section{PROPOSIÇÃO}

Realizar a equivalência de instrumentos de qualidade de vida em saúde bucal com a Classificação Internacional de Funcionalidade, Incapacidade e Saúde (CIF), em pacientes com câncer de boca e outros problemas bucais que buscaram atendimento de urgência. 


\section{MATERIAL E MÉTODOS}

Os pacientes envolvidos no estudo, foram divididos em dois grupos, sendo que o primeiro, com $n=50$ para os pacientes diagnosticados com câncer de cabeça $e$ pescoço (CCP) da Liga de Neoplasias Bucais (LINB), atendidos na Faculdade de Odontologia da Universidade de São Paulo. Os pacientes foram entrevistados na Clínica da faculdade onde estavam no aguardo ou já submetidos ao tratamento odontológico que os levou ao atendimento. Estes pacientes responderam os questionários UW QOL e BCSQ H\&N.

No segundo grupo, os pacientes envolvidos eram os que procuraram o Serviço de Urgências da Faculdade de Odontologia da USP. Com $n=100$, os pacientes foram entrevistados antes ou após o atendimento que os levaram a procurar o Serviço de Urgências da faculdade, independente do motivo da consulta. Os pacientes foram entrevistados na clínica do setor e responderam o questionário Oral Health Impact Profile - (OHIP - 14) (Almeida et al., 2004).

Todos os pacientes atendidos, entre janeiro de 2013 e janeiro de 2014, puderam participar da pesquisa, desde que concordassem - de livre e espontânea vontade - com responder aos questionários: University of Washington - Quality of Life (UW-QOL) (Vartanian et al., 2006), o anexo do Brief Core Set Questionnaire Head and Neck (BCSQ-H\&N) (Rogers et al., 2010) e Oral Health Impact Profile (OHIP - 14) (Almeida et al., 2004). As autorizações estão nos Anexos A, B, C, D e E.

O projeto de pesquisa foi aprovado pelo Comitê de Ética em Pesquisa (CEP) da FOUSP, parecer número 179.710, CAAE número: 10776412.3.0000.0075 (Apêndice A).

Os questionários citados analisam o impacto da condição bucal na qualidade de vida dos pacientes; dois são específicos para câncer de cabeça e pescoço (UWQOL e BCSQ-H\&N) e outro genérico (OHIP-14), todos com possibilidade de aplicação em diversas condições bucais. 
Após a aplicação desses instrumentos, procurou-se associar as dimensões envolvidas com a Classificação Internacional de Funcionalidade, Incapacidade e Saúde (CIF).

Os escores elevados no questionário da Universidade de Washington representam uma condição desejável, ou seja, com menor impacto na condição bucal na qualidade de vida e sua pontuação podendo variar de 0 a 100 pontos.

O questionário BCSQ H\&N foi dividido em três seções. Nas seções 1 e 2, a pontuação, pode variar de 0 a 4 pontos, neste caso indicaria a pior condição possível. Na Seção 3, a pontuação mínima -4 , o que indicaria a pior condição possível; pontuação máxima de +4 , o que indica a melhor condição possível.

A média da pontuação geral dos domínios específicos do Oral Health Impact Profile (OHIP) e os escores elevados, no que se refere a pontuação, representam condições não desejáveis, ou seja, maior impacto da condição bucal na qualidade de vida. A pontuação variou do mínimo possível em cada dimensão: 0; máximo possível em cada dimensão: 8 pontos.

Sendo a pontuação o mínimo possível no escore total: 0; máximo possível no escore total: 56 pontos.

\subsection{Critérios de inclusão}

No caso de pacientes da Liga de Câncer, foram incluídos na pesquisa pacientes que tivessem interesse e disponibilidade em participar da pesquisa e que obedecessem aos quesitos dentro da Classificação Internacional de Doenças (CID10aㅡ Revisão): face interna dos lábios (C00), língua (C01 e C02), gengiva (C03), assoalho de boca (C04), palato e úvula (C05), mucosa oral (C06.0), vestíbulo de boca (C06.1), área retromolar (C06.2), outras partes e partes não especificadas da boca (C06.8, C06.9), amígdala (C09) e orofaringe (C10). Os pacientes assinaram o termo de consentimento livre e esclarecido (Anexo B). Assim, todos os pacientes que foram elegíveis, independentemente da idade, sexo, nível socioeconômico ou cultura, I puderam participar da pesquisa. 
No caso de pacientes do Setor de Urgências da FOUSP, foram convidados a participar os adultos que procuraram pelo Serviço no período da investigação, independentemente da queixa que os levou a buscá-lo.

4.2 Critérios de exclusão

Foram excluídos do projeto pacientes que não se enquadraram nos itens de inclusão na pesquisa, ou que, após convite, recusaram tomar parte no estudo. 


\section{RESULTADOS}

Dos resultados obtidos dos pacientes entrevistados pelo questionário UWQOL na LINB, 50\% dos pacientes eram do gênero masculino e a faixa etária mais acometida pelo câncer foi entre 41 e 60 anos, correspondendo a $48 \%$ da amostra e a localização anatômica mais frequentes foram a língua, palato e laringe. Os domínios responsáveis pelas maiores limitações foram a ansiedade, saliva, paladar, dor e mastigação.

No questionário $\mathrm{BCSQ} \mathrm{H \& N}$ os escores máximo e mínimos não foram alcançados e as categorias da CIF mais frequentes foram b510 (funções de ingestão) e b310 (funções da voz). E os domínios mais pontuados são as relacionadas com as Atividades e Participação.

Dos entrevistados no questionário OHIP, houve um predomínio maior do gênero feminino com até 60 anos de idade e a principal queixa era a dor, o que motivou os pacientes a procurarem pelo serviço. Os domínios mais observados nos pacientes foram dor e desconforto psicológico. 
Tabela 5.1 - Informações sociodemográficas, localização anatômica e tratamentos dos tumores dos pacientes atendidos na Liga de Neoplasias Bucais (LINB) da FOUSP

\begin{tabular}{llcc}
\hline & & $\mathrm{n}$ & $\%$ \\
\hline Gênero & Masculino & 25 & 50 \\
& Feminino & 25 & 50 \\
\hline Faixa etária & até 40 anos & 13 & 26 \\
& de 41 a 60 anos & 24 & 48 \\
& mais de 60 anos & 13 & 26 \\
\hline Localização anatômica & Língua & 14 & 28 \\
& Palato & 8 & 16 \\
& Laringe & 8 & 16 \\
& Assoalho bucal & 5 & 10 \\
& Glândulas salivares & 4 & 8 \\
& Amígdala & 3 & 6 \\
& Lábio & 3 & 6 \\
& Orofaringe & 3 & 6 \\
& Pescoço & 1 & 2 \\
& Outro (linfoma) & 1 & 2 \\
\hline Tratamento realizado & Cirurgia & 3 & 6 \\
& Quimioterapia & 2 & 4 \\
& Cirurgia e Quimioterapia & 2 & 4 \\
& Cirurgia e Radioterapia & 6 & 12 \\
& Quimioterapia & 12 & 24 \\
& Radioterapia & & \\
& Cirurgia, Quimioterapia e & 25 & 50 \\
& Radioterapia & & \\
\hline & & &
\end{tabular}

A tabela 5.1 apresenta as informações sociodemográficas dos pacientes atendidos na Liga de Neoplasias Bucais (LINB). Metade deles do gênero masculino. A faixa etária mais frequente ficou entre 41 e 60 anos de idade, o que corresponde a $48 \%$ da amostra.

Com relação à localização anatômica, língua, palato e laringe foram as mais frequentes: $28 \%, 16 \%$ e $16 \%$ dos casos, respectivamente (Tabela 5.1 ). 
Tabela 5.2 - Média da pontuação geral e segundo domínios específicos do University of Washington Quality of Life (UW-QoL) dos pacientes atendidos na Liga de Neoplasias Bucais (LINB) da FOUSP

\begin{tabular}{lc}
\hline Dimensões & pontos $^{*}$ \\
\hline Ansiedade & 48,6 \\
Saliva & 49,9 \\
Paladar & 66,0 \\
Dor & 68,0 \\
Mastigação & 70,0 \\
Deglutição & 71,4 \\
Humor & 72,5 \\
Aparência & 75,5 \\
Recreação & 77,0 \\
Fala & 78,8 \\
Atividade & 79,0 \\
Ombros & 80,0 \\
\hline Total & 69,7 \\
\hline
\end{tabular}

A tabela 5.2 mostra os escores alcançados pelos domínios do questionário UW-QOL. Do total de 100 pontos possíveis, a média foi 69,7 pontos. Os domínios responsáveis pelas maiores limitações dos pacientes foram: ansiedade, saliva, paladar, dor e mastigação. 
Tabela 5.3 - Média da pontuação geral e segundo domínios específicos da Seção 1 do Brief Core Set Questionnaire Head and Neck - (BCSQ-H\&N) dos pacientes atendidos na Liga de Neoplasias Bucais (LINB) da FOUSP

\begin{tabular}{lc}
\hline Seção 1: Problemas com partes do seu corpo & $\begin{array}{c}\text { Média de } \\
\text { pontos* }\end{array}$ \\
\hline 1. Em relação a função da boca em geral & 1,84 \\
a. Mordida & 0,72 \\
b. Mastigação & 0,76 \\
c. Movendo comida na boca & 1,74 \\
d. Salivação & 1,74 \\
e. Engolir & 1,56 \\
f. Sucção & 1,26 \\
2. Função da voz em geral & 1,76 \\
a. Som que produz & 0,58 \\
b. Qualidade do som & 0,78 \\
3. Função Emocional & 1,34 \\
4. Energia e condução (Motivação) & 1,26 \\
5. Respiração (Inspirar ou Expirar) & 0,22 \\
6.Estrutura da boca em geral & 1,54 \\
a. Estrutura dos dentes & 1,06 \\
b. Estrutura dos lábios & 0,6 \\
c. Estrutura da língua & 1,08 \\
d. Palato & 0,5 \\
e. Estrutura de outras partes da boca & 0,96 \\
7. Estrutura de sua garganta & 0,92 \\
8. Estrutura de sua caixa de voz & 0,28 \\
9. Estrutura de outras partes de sua cabeça e pescoço & 0,92 \\
10. Dor & 1,1 \\
\hline
\end{tabular}

Tabela 5.4 - Média da pontuação geral e segundo domínios específicos da Seção 2 do Brief Core Set Questionnaire Head and Neck - (BCSQ-H\&N) dos pacientes atendidos na Liga de Neoplasias Bucais (LINB) da FOUSP

\begin{tabular}{lc}
\hline Seção 2: Problemas com a atividade social e funcionamento & $\begin{array}{c}\text { Média de } \\
\text { pontos }\end{array}$ \\
\hline 1. Falando & 0,60 \\
2. Bebendo & 0,46 \\
3. Comendo & 1,10 \\
4. Realizar sua rotina diária & 1,18 \\
5. Apoiando-se financeiramente & 1,18 \\
6. Relacionamento familiares & 0,63 \\
\hline
\end{tabular}


Tabela 5.5 - Média da pontuação geral e segundo domínios específicos da Seção 3 do Brief Core Set Questionnaire Head and Neck - (BCSQ-H\&N) dos pacientes atendidos na Liga de Neoplasias Bucais (LINB) da FOUSP

\begin{tabular}{lc}
\hline Seção 3: Problemas com o ambiente & $\begin{array}{c}\text { Média de } \\
\text { pontos }^{*}\end{array}$ \\
\hline $\begin{array}{l}\text { 1. Quanto sua família tem ajudado ou sido um obstáculo? } \\
\text { 2. Quanto os profissionais de saúde envolvidos no seu }\end{array}$ & 3,46 \\
$\begin{array}{l}\text { atendimento têm sido uma ajuda ou um obstáculo? } \\
\text { 3. Quanto os alimentos (sólidos), líquidos, vitaminas etc., que }\end{array}$ & 1,82 \\
$\begin{array}{l}\text { você consome, são um problema ou não? } \\
\begin{array}{l}\text { 4. Quanto de uma ajuda ou obstáculo seus medicamentos } \\
\text { (prescritos ou comprados de balcão)? }\end{array}\end{array}$ & 2,60 \\
\hline
\end{tabular}

Nas tabelas 5.3, 5.4 e 5.5 - referentes respectivamente às seções 1,2 e 3 do questionário BCSQ H\&N - não foram observadas pontuação mínima nem a máxima do escore. A categoria mais frequente na tabela 5.3 Seção 1 , foi b510 (funções de ingestão) e b310 (funções da voz).

A tabela 5.4 demonstra que categorias mais frequentes são relacionadas com as Atividades e Participação, d230 (rotina diária), d870 (ajuda financeira) e d550 (comer).

$\mathrm{Na}$ tabela 5.5, as categorias da CIF que refletem os profissionais de saúde envolvidos: atendimento (e355) e relacionamento com familiares (e310), foram as categorias mais pontuadas. 
Tabela 5.6 - Informações sócio-demográficas e motivo da consulta dos pacientes atendidos pelo Setor de Urgências da FOUSP

\begin{tabular}{llcc}
\hline & & $\mathrm{n}$ & $\%$ \\
\hline Gênero & Masculino & 45 & 45 \\
& Feminino & 55 & 55 \\
\hline Faixa etária & até 40 anos & 45 & 45 \\
& de 41 a 60 anos & 47 & 47 \\
& mais de 60 anos & 8 & 8 \\
\hline Motivo da consulta & Dor & 81 & 81 \\
& Fratura /trauma dentário & 6 & 6 \\
& Queda de prótese & 2 & 2 \\
& Sangramento & 2 & 2 \\
& Outros & 9 & 9 \\
\hline
\end{tabular}

A tabela 5.6 apresenta informações sociodemográficas e o motivo da consulta dos pacientes atendidos no Setor de Urgências da FOUSP. Houve predominância do gênero feminino (55\%), de pacientes com até 60 anos (92\%) e dor como principal queixa que levou os pacientes a procurarem pelo Serviço (81\%).

Tabela 5.7 - Média da pontuação geral e segundo domínios específicos do Oral Health Impact Profile (OHIP) dos pacientes atendidos pelo Setor de Urgências da FOUSP

\begin{tabular}{lc}
\hline Dimensões & pontos $^{*}$ \\
\hline Dor física & 5,34 \\
Desconforto psicológico & 5,00 \\
Incapacidade física & 4,00 \\
Incapacidade psicológica & 3,19 \\
Incapacidade social & 2,68 \\
Deficiência & 2,30 \\
Limitação funcional & 2,15 \\
\hline Total & 24,88 \\
\hline
\end{tabular}

A tabela 5.7 mostra a média de pontuação do OHIP-14 dos pacientes atendidos no Setor de Urgências. Os pacientes apresentam escore médio de 24,88 pontos, num total de 56 pontos possíveis. Os impactos mais presentes foram dor física e desconforto psicológico. 
Tabela 5.8 - Categorias da CIF identificadas nas questões do questionário UW - QOL

\begin{tabular}{|c|c|c|}
\hline Tópico & $\begin{array}{l}\text { Questão nr } \\
\text { UW QoL }\end{array}$ & Categorias da CIF \\
\hline Dor, & 1 & $\begin{array}{l}\text { b280 - Sensação de dor } \\
\text { e110 - Produtos ou substâncias para }\end{array}$ \\
\hline Medicação & & $\begin{array}{l}\text { consumo pessoal } \\
\text { e1101- Medicamentos }\end{array}$ \\
\hline Aparência & 2 & $\begin{array}{l}\text { b180 - Funções de experiência pessoal e } \\
\text { do tempo }\end{array}$ \\
\hline Imagem Corporal & & b1801 - Imagem do corpo \\
\hline Nível de atividade & 3 & $\begin{array}{l}\text { d910 - Vida comunitária } \\
\text { d460 - Deslocar-se por diferentes locais }\end{array}$ \\
\hline Tempo de Lazer & 4 & d 920 - Recreação e lazer \\
\hline Deglutição & 5 & $\begin{array}{l}\text { b510 - Função de Ingestão } \\
\text { b5105 - Deglutição }\end{array}$ \\
\hline Mastigar & 6 & $\begin{array}{l}\text { b510 - Função de Ingestão } \\
\text { b5102 - Mastigar }\end{array}$ \\
\hline Fala & 7 & $\begin{array}{l}\text { b310 - Funções da voz } \\
\text { b3100 - Produção da voz } \\
\text { b3101 - Qualidade de voz }\end{array}$ \\
\hline Ombro & 8 & s720 - Estrutura da região do ombro \\
\hline Paladar & 9 & b250 - Função gustativa \\
\hline Saliva & 10 & $\begin{array}{l}\text { b510 - Função de Ingestão } \\
\text { b5104 - Salivação }\end{array}$ \\
\hline Humor & 11 & $\begin{array}{l}\text { b126 - Funções do temperamento e da } \\
\text { personalidade }\end{array}$ \\
\hline Ansiedade & 12 & b152 - Funções emocionais \\
\hline
\end{tabular}

A tabela 5.8 mostra as categorias da Classificação Internacional de Funcionalidade, Incapacidade e Saúde (CIF) das questões abordadas pelo UWQOL. Diversas categorias da CIF apresentaram-se como importantes no dia a dia dos pacientes entrevistados. Assim, cita-se a categoria b1801 (Imagem do corpo), já que a aparência afetou grandemente o cotidiano deles, muitos dos quais relataram que as atividades de recreação e lazer (d920) foram fortemente afetadas pela condição bucal. Essa tabela apresenta ainda dificuldade de mastigação (b5102), deglutição (b51050), fala (b320), conversação (d350); enfim, tanto as atividades de interação com outras pessoas, quanto as funcionais são mencionadas no relato dos pacientes que responderam a questionário UW-QOL e foram categorizados na CIF. 
Tabela 5.9 - Categorias da CIF identificadas no questionário OHIP - 14

\begin{tabular}{|c|c|c|}
\hline Tópicos & $\begin{array}{l}\text { Questão } \\
\text { OHIP - } 14\end{array}$ & Categorias da CIF \\
\hline Fala & 1 & b310 - Função da Voz \\
\hline Paladar & 2 & b250 - Função gustativa \\
\hline Dor & 3 & $\begin{array}{l}\text { b280 - Sensação de dor } \\
\text { s320 - Estrutura da boca }\end{array}$ \\
\hline Mastigação & 4 & $\begin{array}{l}\text { b510 - Funções de Ingestão } \\
\text { b5102 - Mastigar } \\
\text { b126 - Funções do temperamento } \\
\text { e da personalidade }\end{array}$ \\
\hline Ansiedade & 5 & b152 - Funções emocionais \\
\hline Humor & 6 & $\begin{array}{l}\text { b126 - Funções do temperamento } \\
\text { e da personalidade }\end{array}$ \\
\hline $\begin{array}{l}\text { Mastigação e } \\
\text { Deglutição }\end{array}$ & 7 & $\begin{array}{l}\text { b510 - Função de Ingestão } \\
\text { b5105 - Deglutição }\end{array}$ \\
\hline $\begin{array}{l}\text { Mastigar/ } \\
\text { Alimentos }\end{array}$ & 8 & $\begin{array}{l}\text { b510 - Função de Ingestão } \\
\text { e110 - Produtos ou substâncias } \\
\text { para consumo pessoal } \\
\text { e1100 - Alimentos }\end{array}$ \\
\hline Repouso & 9 & b 134 - Funções do Sono \\
\hline Personalidade & 10 & $\begin{array}{l}\text { b126 - Funções do temperamento } \\
\text { e da personalidade }\end{array}$ \\
\hline Temperamento & 11 & b152 - Funções emocionais \\
\hline Atividade & 12 & d230 - Realizar a rotina diária \\
\hline Nível de Atividade & 13 & b117 - Funções intelectuais \\
\hline Atividade & 14 & d230 - Realizar a rotina diária \\
\hline
\end{tabular}

A tabela 5.9 descreve as categorias da Classificação Internacional de Funcionalidade, Incapacidade e Saúde (CIF) do questionário Oral Health Impact Profile (OHIP-14). Diversas categorias da CIF foram identificadas nas respostas obtidas pelos pacientes entrevistados. A categoria dor (b280) foi a mais obtida em se tratando de Serviço de Urgência, porém deve-se salientar que não é menos importante o motivo de uma categoria afetar outra (d920 - incapacidade social). A tabela 5.9, mostra, ainda, outras categorias, tais como realizar a rotina diária (d230), alterações de humor (b1520) e outras mais específicas, mastigação (b5102), deglutição (b5105), como causadoras de impactos. 
Tabela 5.10 - Frequência das categorias da CIF, segundo aparecimento nas respostas dos questionários OHIP-14, UW-QOL e BCSQ H\&N

\begin{tabular}{|c|c|c|c|c|c|}
\hline & 1 & 2 & 3 & 4 & 5 \\
\hline $\begin{array}{l}\text { b280 } \\
\text { Sensação de dor }\end{array}$ & OHIP & & & UW & BCSQ \\
\hline $\begin{array}{l}\text { b152 } \\
\text { Ansiedade } \\
\text { Funções emocionais }\end{array}$ & UW & & & BCSQ & OHIP \\
\hline $\begin{array}{l}\text { b310 } \\
\text { Função da voz }\end{array}$ & & BCSQ & & & \\
\hline $\begin{array}{l}\text { b152 } \\
\text { Humor } \\
\text { Funções emocionais }\end{array}$ & & OHIP & BCSQ & OHIP & \\
\hline $\begin{array}{l}\text { b130 } \\
\text { Atividade }\end{array}$ & & & OHIP & & \\
\hline $\begin{array}{l}\text { b510 } \\
\text { Funções da boca }\end{array}$ & $\begin{array}{l}\text { BCSQ } \\
\text { geral }\end{array}$ & $\begin{array}{l}\text { UW } \\
\text { saliva }\end{array}$ & & & $\begin{array}{c}\text { UW } \\
\text { deglutição }\end{array}$ \\
\hline $\begin{array}{l}\text { b250 } \\
\text { Paladar }\end{array}$ & & & UW & & \\
\hline
\end{tabular}

A tabela 5.10 demonstra as categorias mais presentes da CIF, segundo frequência de aparecimento nas respostas dos questionários OHIP-14, UW-QOL e BCSQ H\&N. As categorias da CIF, observadas em todos os questionários aplicados e dispostos em ordem de aparecimento foram: Funções do Corpo, representadas na tabela 5.10 pela letra "B" (body) em inglês. No questionário OHIP-14 a categoria mais frequente foi a b280 (sensação de dor); no questionário UW-QOL, a b152 (ansiedade - fatores emocionais); e, no questionário BCSQ H\&N, a categoria mais presente foi b510 (funções da boca). 


\section{DISCUSSÃO}

A avaliação da qualidade de vida é tarefa complexa, que envolve questões, tanto gerais, quanto específicas, sobre os diferentes domínios (dor, mastigação, fala entre outras) e podem ser avaliadas por diferentes grupos de classificações clínicas e sociodemográficas.

Quanto à pontuação de QV, as características sociodemográficas, de modo geral, apresentaram alteração em relação à estimativa de Câncer no Brasil (INCA, 2014) na proporção 3:1, respectivamente para homens e mulheres, para o câncer; e, na amostra dos entrevistados, $50 \%$.

Foi observado, na análise dos resultados, que os cinco domínios mais pontuados na aplicação do questionário UW-QOL foram: ansiedade, saliva, paladar, dor e mastigação. Radford et al. (2004), ao aplicarem o UW-QOL em pacientes do Hospital da Cidade em Birmingham (Reino Unido), observaram que em $70 \%$ dos casos de câncer de cabeça e pescoço, os escores aumentaram para ansiedade, dor e humor. A maior expectativa dos pacientes era em relação ao câncer e seu prognóstico, e também aos domínios funcionais que contribuem para o relacionamento social.

De modo geral, os pacientes diagnosticados com tumores localizados na região bucal, apresentaram preocupação com: aparência, mastigação, deglutição, fala e salivação, para os casos de pacientes irradiados. McDonough et al. (1996) avaliaram as mudanças nos níveis de QV, nas diversas fases de tratamento; a fase cirúrgica alterou de forma significativa essa questão, com forte piora na avaliação de ansiedade e relacionamento social, o que alterou a QV desses pacientes.

Da análise da pontuação obtida nos escores do questionário UW-QOL, sendo a pior pontuação para 48,6 pontos numa escala de 0 pontos (pior pontuação) e 100 pontos (melhor pontuação), os resultados traduzem em pacientes bem tratados pelos hospitais ou centros de tratamentos para pacientes com câncer de cabeça e pescoço, independente do tratamento realizado, seja 
cirurgia, quimioterapia e radioterapia ou uma combinação delas. Fato que comprova o bom atendimento e a melhora no prognóstico do paciente e consequentemente melhora na qualidade de vida

Apesar de os instrumentos de QV (UW-QOL e OHIP-14) abordarem situações e problemas diferentes, no questionário UW-QOL, o domínio "dor" não foi o mais pontuado, em pacientes irradiados, tratados cirurgicamente e, às vezes, submetidos a tratamentos com margem de segurança e cirurgias de grande porte.

O questionário $B C S Q-H \& N$ foi considerado rápido para completar e apresentou, como vantagem, ser questionário curto, o que facilita a entrevista para $\mathrm{o}$ paciente (Rogers et al., 2010), embora não aborde algumas categorias da CIF de interesse, tais como: gosto (b250), abertura de boca (s7103), função articulação (b320), estrutura de região dos ombros (s720), categorias já presentes no questionário UW-QOL.

No questionário OHIP-14, os domínios que apresentaram maior pontuação da análise dos resultados foram dor e desconforto psicológico. Brennan et al. (2006) aplicaram, em pacientes do Centro Australiano de Pesquisa de População e Saúde Oral da Faculdade de Odontologia da Universidade de Adelaide (Austrália do Sul, Austrália), e encontraram como principal queixa a dor física e o desconforto psicológico, na maioria das respostas.

Tendo em vista os diversos e diferentes domínios afetados, para cada instrumento de qualidade de vida, sublinha-se a importância do acompanhamento dos pacientes por cirurgião-dentista, independentemente das fases do tratamento e da patologia. O dentista, em conjunto com a equipe multidisciplinar, deve reconhecer, tratar, preparar e acompanhar o paciente, por meio de análise em conjunto, pelos prognósticos a partir dos quais elabora propostas de tratamento e reabilitação. Além disso, o tratamento posterior é muito importante para a saúde bucal e contribui muito para melhora da QV. Uma vez incorporada a CIF no SUS, conforme aprovação do CNS-MS (Resolução n 452 de 10 maio 2012) (Brasil, 2012), sua utilização em grande escala deve estimular ao mesmo tempo a crescente discussão sobre os níveis de QV, não somente com a finalidade de garantir benefício ou aposentadoria por invalidez. 
Os domínios que alteram os níveis de QV não sofrem significativas alterações em tratamentos menores e menos invasivos, por não comprometer a aparência, as funções bucais nem a comunicação social. Mas pacientes com tratamentos concluídos, tem um prognóstico e uma melhora em sua qualidade de vida, talvez pela expectativa que o tratamento proporciona ao paciente em relação ao cancer de cabeça e pescoço.

Os dados, obtidos no questionário OHIP-14 para os pacientes atendidos na Clínica de Urgências, mostram intensa preocupação com a dor física, mesmo que associado a edema facial, pois muitas vezes resulta deformidade facial o que altera a aparência. Porém a melhora foi comprovada na pontuação dos escores obtidos pela resolução dos tratamentos realizados na Clínica de Urgência que poderiam varia de 0 pontos (melhor condição) a 8 pontos (pior condição) e o domínio mais pontuado foi a dor física com escore de 5,34 pontos.

Os pacientes da LINB, que participaram do estudo, demonstraram mais preocupação com a ansiedade em relação ao câncer, ao prognóstico e às funções bucais (fala, mastigação, deglutição, salivação, aparência), domínios estes que contribuem diretamente para a interação social.

A utilização da CIF pode contribuir de forma positiva para o estabelecimento de políticas públicas voltadas para as pessoas com deficiência ou incapacidade. A CIF foi incluída, mesmo antes da sua edição, como padrão para caracterização da deficiência na proposta do Estatuto da Pessoa com Deficiência, apresentada em consulta pública ao Senado Federal, embora essa menção tenha desaparecido e reaparecido em minutas subsequentes. Este pode ser o reflexo da grande expectativa com a qual é aguardada, no Brasil, a utilização da CIF em Português, em especial pelas organizações de pessoas com deficiências e instituições relacionadas.

A CIF, ao ser usada de forma mais ampla ou mais consistente, permitirá que se adote nova terminologia, que se padronizem conceitos e que se reflita sobre o tema, ou seja, pode contribuir para tornar mais claro o uso das palavras, para propiciar entendimento e comunicação mais uniforme entre as pessoas interessadas nessa área; assim, será possível alcançar as vantagens da terminologia padronizada. 
É importante que o poder público e os formuladores de políticas participem da discussão para a obtenção de definições e avaliações mais claras e mais justas da deficiência ou incapacidade.

A CIF é propriedade de todos os seus usuários. A sociedade interessada, em especial as pessoas com deficiência, as organizações sociais e os formuladores de políticas, devem se apropriar do conhecimento sobre os usos potenciais da CIF e CID. O uso dessas classificações como instrumentos pode contribuir para que as reais condições de vida das pessoas com deficiência venham a fazer parte das estatísticas, permitindo guiar ações e decisões, delinear políticas, definir intervenções e destinar orçamentos entre outras. 


\section{CONCLUSÕES}

Foi observada equivalência entre os domínios dos instrumentos de qualidade de vida e a CIF. Assim, a CIF constitui instrumento útil para avaliar a real situação de saúde do paciente, além dos aspectos clínicos. 


\section{REFERÊNCIAS 1}

Almeida AA, Loureiro CA, Araújo VE. Um estudo transcultural de valores de saúde bucal utilizando o instrumento OHIP-14 (Oral Health Impact Profile) na Forma Simplificada. Parte I: Adaptação cultural e linguística. UFES Rev Odontol. 2004;6(1):6-15.

Biazevic MGH, Antunes JLF, Togni J, Andrade FPA, Carvalho MB, Wünsch-Filho V. Immediate impact of primary surgery on health-related quality of life of hospitalized patients with oral and oropharyngeal cancer. J Oral Maxillofac Surg. 2008 Jul;66(7):1343-50.

Biazevic MGH, Michel Crosato E, lagher F, Pooter CE, Correa SL, Grasel CE. Impact of oral health on quality of life among the elderly population of Joaçaba, Santa Catarina, Brazil. Braz Oral Res. 2004 Jan/Mar;18(1):85-91.

Bowling A, Brazier J. Quality of life in social science and medicine - introduction. Soc Sci Med. 1995;41:1337-8.

Brasil. Ministério da Saúde. Conselho Nacional de Saúde - Resolução n 452 de 10 maio 2012. [Brasília: Ministério da Saúde; 2012] [citado 23 ago. 2014]. Disponível em: http://bvsms.saude.gov.br/bvs/saudelegis/cns/2012/res0452_10_05_2012.html.

Brennan DS, Singh KA, Spencer AJ, Roberts-Thomson KF. Positive and negative affect and oral health-related quality of life. Health Qual Life Outcomes. 2006;4:83.

Casado D. Conceptos sobre la Discapacidad. Bol Real Patronato Discapacidad. 2001;50:5-13.

Cieza A, Brockow T, Ewert T, Amman E, Kollerits B, Chatterji S, et al. Linking health - status measurements to the international classification of functioning, disability and health. J Rehabil Med. 2002 Sept;34(5):205-10.

Cohen-Carneiro F, Souza-Santos R, Rebelo MA. Quality of life related to oral health: contribution from social factors. Ciên Saúde Colet. 2011;16(Suppl 1):1007-15.

\footnotetext{
${ }^{1}$ De acordo com Estilo Vancouver.
} 
Cremonese C, Backes V, Olinto MTA, Dias-da-Costa JS, Pattussi MP. Neighborhood sociodemographic and environmental contexts and self-rated health among Brazilian adults: a multilevel study. Cad Saúde Pública. 2010 Dec;26(12):2368-78.

Di Nubila HBV, Buchalla CM. O papel das classificações da OMS - CID e CIF nas definições de deficiência e incapacidade. Rev Bras Epidemiol. 2008 jun;11(2):32435.

Egea C, Sarabia A. Clasificaciones de la OMS sobre discapacidad. Bol Real Patronato Discapacidad. 2001;50:15-30.

Gabardo MCL, Moysés ST, Moysés S. Autopercepção de saúde bucal conforme o Perfil de Impacto da Saúde Bucal (OHIP) e fatores associados: revisão sistemática. Rev Panam Salud Publica. 2013 jun;33(6):439-45.

Hammerlid E, Bjordal K, Ahlner-Elmqvist M, Boysen M, Evensen JF, Biorklund A, et al. A prospective study of quality of life in head and neck cancer patients. Part I: At diagnosis. Laryngoscope. 2001 Apr;111(4 Pt 1):669-80.

Hassan SJ, Weymuller EA JR. Assessment of quality of life in head and neck cancer patients. Head Neck. 1993 Nov-Dec;15(6):485-96.

INCA - Instituto Nacional de Câncer José Alencar Gomes da Silva. Coordenação de Prevenção e Vigilância. Estimativa 2014: incidência de câncer no Brasil. Rio de Janeiro: INCA; 2014.

Ikebe K, Watkins CA, Ettinger RL, Sajima H, Nokubi T. Application of short-form oral health impact profile on elderly Japanese. Gerodontology. 2004 Sept;21(3):167-76.

Laurenti R. Análise da informação em saúde: 1893-1993, cem anos da Classificação Internacional de Doenças. Rev Saúde Pública. 1991 dez;25(6):407-17.

Leal CMS. Reavaliar o conceito da qualidade de vida. Portugal: Universidade dos Açores; 2008. Projeto de Pesquisa.

Locker D, Gibson B. Discrepancies between self-ratings of and satisfaction with oral health in two older adult populations. Community Dent Oral Epidemiol. 2005 Aug;33(4):280-8. 
Lundegren N, Axtelius B, Håkansson J, Akerman S. Dental treatment need among 20 to 25-year-old Swedes: discrepancy between subjective and objective need. Acta Odontol Scand. 2004 Apr;62(2):91-6.

McDonough E, Varvares MA, Dunphy FR, Dunleavy T, Dunphy CH, Boyd JH. Changes in quality-of-life scores in a population of patients treated for squamous cell carcinoma of the head and neck. Head Neck 1996 Nov-Dec;18(6):487-93.

Miettinem OS. Quality of life from the epidemiologic perspective. J Chronic Dis. 1987;40(6):641-3.

Miotto MHMB, Barcellos LA, Velten DB. Avaliação do impacto na qualidade de vida causado por problemas bucais na população adulta e idosa em município da Região Sudeste. Ciênc Saúde Coletiva. 2012 fev.;17(2):397-406.

Murphy BA, Ridner S, Wells N, Dietrich M. Quality of life research in head and neck cancer: a review of current state of the science. Crit Rev Oncol Hematol. 2007 Jun;62(3):251-67.

Nguyen TC, Witter DJ, Bronkhorst EM, Gerritsen AE, Creugers NHJ. Chewing ability and dental functional status. Int J Prosthodont. 2011 Sept-Oct;24(5):428-36.

Nobre MRC. Qualidade de vida. Arq Bras Cardiol. 1995;64(4):299-300.

Organização Mundial da Saúde. CIF: Classificação Internacional de Funcionalidade, Incapacidade e Saúde. Centro Colaborador da Organização Mundial da Saúde para a Família de Classificações Internacionais, tradutor. São Paulo: EDUSP; 2003.

Organização Mundial da Saúde. Classificação Estatística Internacional de Doenças e Problemas Relacionados à Saúde: CID-10 Décima revisão. 3a ed. Centro Colaborador da OMS para a Classificação de Doenças em Português, tradutor. São Paulo: EDUSP; 1996.

Organização Mundial da Saúde. Classificação Internacional das Deficiências, Incapacidades e Desvantagens (handicaps): um manual de classificação das conseqüências das doenças. Lisboa: OMS; 1989.

Radford K, Woods H, Lowe D, Rogers SN. A UK multi-centre pilot study of speech and swalloing outcomes following head and neck cancer. Clin Otolaryngol Allied Sci. 2004 Aug;29(4):376-81. 
Reisine ST. Theoretical considerations in formulating sociodental indicators. Soc Sci Med A. 1981 Dec;15(6):745-50.

Rogers SN, Forgie S, Lowe D, Precious L, Haran S, Tschiesner U. Development of the International Classification of Functioning, Disability and Health as a brief head and neck cancer patient questionnaire. Int J Oral Maxillofac Surg. 2010 Oct;39(10):975-82.

Rogers SN, Laher SH, Overend L, Lowe D. Importance - rating using the University of Washington Quality of Life questionnaire in patients treated by primary surgery for oral and oro-pharyngeal cancer. J Craniomaxillofac Surg. 2002 Apr;30(2):125-32.

Rogers SN, Lowe D, Brown JS, Vaughan ED. A comparison between the University of Washington Head and Neck Disease-Specific measure and the Medical Short Form 36, EORTC QOQ-C33 and EORTC Head and Neck 35. Oral Oncol. 1998 Sept;34(5):361-72.

Rogers SN, Lowe D, Brown JS, Vaughan ED. The University of Washington head and neck cancer measure as a predictor of outcome following primary surgery for oral cancer. Head Neck. 1999 Aug;21(5):394-401.

Rogers SN, Lowe D, Fisher SE, Brown JS, Vaughan ED. Health- related quality of life and clinical function after primary surgery for oral cancer. $\mathrm{Br} \mathrm{J}$ Oral Maxillofac Surg. 2002 Feb;40(1):11-8.

Rogers SN, Lowe D, Humphris G. Distinct patient groups in oral cancer: a prospective study of perceived health status following primary surgery. Oral Oncol. 2000 Nov;36(6):529-38.

Rogerson RJ. Environmental and health-related quality of life: conceptual and methodological similarities. Soc Sci Med. 1995 Nov;41(10):1373-82.

Sayed SI, Elmiyeh B, Rhys-Evans P, Syrigos KN, Nutting CM, Harrington KJ, et al. Quality of life and outcomes research in head and neck cancer: a review of the state of the discipline and likely future directions. Cancer Treat Rev. 2009;35(5):397-402.

Sheiham A, Alexander D, Cohen L, Marinho V, Moysés S, Petersen PE, et al. Global oral health inequalities: task group-implementation and delivery of oral health strategies. Adv Dent Res. 2011 May;23(2):259-67. 
Slade GD. Derivation and validation of a short-form oral health impact profile. Community Dent Oral Epidemiol. 1997 Aug;25(4):284-90.

Slade GD, Spencer AJ. Development and evaluation of the oral Health Impact Profile. Community Dent Health. 1994 Mar;11(1):3-11.

Testa MA, Simonson DC. Assessment of quality-of-life outcomes. N Engl J Med. 1996 Mar;334(13):835-40.

The WHOQOL Group. The development of the World Health Organization quality of life assessment instrument (the WHOQOL). In: Orley J, Kuyken W, editors. Quality of life assessment: international perspective. Heidelberg: Springerg; 1994. p. 41-60.

Tschiesner U, Linseisen E, Coenen M, Rogers S, Harreus U, Berghaus A, et al. Evaluating sequelae after head and neck cancer from the patient perspective with the help of the International Classification of Functioning, Disability and Health. Eur Arch Otorhinolaryngol. 2009a Mar;266(3):425-36.

Tschiesner U, Rogers SN, Harreus U, Berghaus A, Cieza A. Comparison of outcome measures in head and neck cancer - a literature review 2000-2006. Head Neck 2009b Feb;31(2):251-9.

Tschiesner U, Rogers SN, Harreus U, Berghaus A, Cieza A. Content comparison of quality of life questionnaires used in Head and Neck Cancer based on the International Classification of Functioning, Disability and Health (ICF): a systematic review. Eur Arch Otorhinolaryngol. 2008 Jun;265(6):627-37.

Tschiesner UM, Chen A, Funk G, Yueh B, Rogers SN. Shortfalls in international, multidisciplinary outcome data collection following head and neck cancer: does the ICF Core Set for HNC provide a common solution? Oral Oncol. 2009c, Oct;45(10):849-55.

Tugwell P, Bennett KJ, Sackett D, Haynes B. Relative risks, benefits and costs of intervention. In: Warren KS, Mahmoud AAF, editores. Tropical and Geographical Medicine. 2a ed. New York: McGraw-Hills; 1985. p. 1097-113.

Üstun TB. The International Classification of Functioning, Disability and Health: a common framework for describing health states. In: Murray CJL, Solomon JA, Mathers CD, Lopez AD, editors. Summary Measures of Population Health: concepts, ethics, measurement and applications. Geneva: WHO; 2002. p. 344-8. 
Vartanian JG, Carvalho AL, Yueh B, Furia CL, Toyota J, McDowell JA, et al. Brazilian-Portuguese validation of the University of Washington Quality of Life Questionnaire for patients with head and neck cancer. Head Neck. 2006 Dec;28(12):1115-21.

Vartanian JG, Carvalho AL, Yueh B, Priante AVM, Melo RLM, Correia LM, et al. Long term quality of life evaluation after head and neack cancer treatment in a developing country. Arch Otolaryngol Head Neack Surg. 2004 Oct;130(10):1209-13.

Vido MB, Fernandes RAQ. Qualidade de vida: considerações sobre conceitos e instrumentos de medida. Braz J Nurs [online]. 2007;6(2) [citado 27 out. 2011]. Disponível em: <http://www.uff.br/objnursing/index.php/nursing/rt/ printerFriendly/j.1676-4285.2007.870/197>

Walter MH, Woronuk JI, Tan HK, Lenz U, Koch R, Boening KW, et al. Oral health related quality of life and its association with sociodemographic and clinical findings in 3 northern outreach clinics. J Can Dent Assoc. 2007 Mar;73(2):153.

Weymuller EA JR, Alsarraf R, Yueh B, Deleyiannis FW, Coltrera MD. Analysis of the performance characteristics of the University of Washington Quality of Life instrument and its modification (UW-QOL-R). Arch Otolaryngol Head Neck Surg. 2001 Mar;127(5):489-93.

World Health Organization. Family Development Committee. Implications for the ICD of the ICF. Meeting of Heads of WHO Collaborating Centres for the Family of International Classifications. Bethesda: World Health Organization; 2001. (WHO/GPE/CAS/C/01.55). 
APÊNDICE A - Parecer Consubstanciado do CEP

\section{FACULDADE DE ODONTOLOGIA DA UNIVERSIDADE DE SÃO}

\section{PARECER CONSUBSTANCIADO DO CEP}

\section{DADOS DO PROJETO DE PESQUISA}

Título da Pesquisa: EQUIVALÊNCIA DE INSTRUMENTOS DE QUALIDADE DE VIDA EM SAÚDE BUCAL COM A CLASSIFICAÇÃO INTERNACIONAL DE FUNCIONALIDADE, INCAPACIDADE E SAÚDE

Pesquisador: Maria Gabriela Haye Biazevic

Área Temática:

Versão: 1

CAAE: 10776412.3 .0000 .0075

Instituição Proponente: Universidade de Sao Paulo

Patrocinador Principal: Financiamento Próprio

\section{DADOS DA NOTIFICAÇÃO}

Tipo de Notificação: Envio de Relatório Parcial

Detalhe:

Justificativa: Relatório sobre o andamento da pesquisa.

Data do Envio: 05/08/2013

Situação da Notificação: Aguardando revisão do parecer do colegiado

\section{DADOS DO PARECER}

Número do Parecer: 389.963

Data da Relatoria: 21/08/2013

Apresentação da Notificação:

Relatorio parcial apresentado.

Objetivo da Notificação:

Apresentar relatorio parcial

Avaliação dos Riscos e Benefícios:

$\mathrm{Ndn}$

Comentários e Considerações sobre a Notificação:

O projeto esta seguindo cronograma

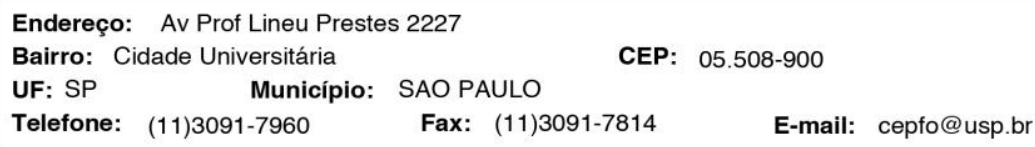




\section{FACULDADE DE ODONTOLOGIA DA UNIVERSIDADE DE SÃO}

Continuação do Parecer: 389.963

Considerações sobre os Termos de apresentação obrigatória:

Nao se aplica

Recomendações:

Nfnd

Conclusões ou Pendências e Lista de Inadequações:

Seguindo o cronograma e apresentar relatorio em seis meses.

Situação do Parecer:

Aprovado

Necessita Apreciação da CONEP:

Não

Considerações Finais a critério do CEP:

SAO PAULO, 10 de Setembro de 2013

Assinador por:

Décio dos Santos Pinto Junior

(Coordenador)

Endereço: Av Prof Lineu Prestes 2227

Bairro: Cidade Universitária

UF: SP Município: SAO PAULO

Telefone: (11)3091-7960

Fax: (11)3091-7814

E-mail: cepfo@usp.br 
ANEXO A - Autorização de Pesquisa na Clínica Odontológica

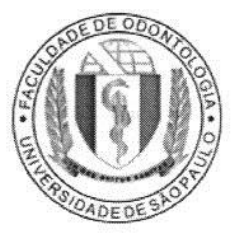

\author{
UNIVERSIDADE DE SÃO PAULO \\ FACULDADE DE ODONTOLOGIA \\ CLÍNICA ODONTOLÓGICA
}

\title{
AUTORIZAÇÃO DE PESQUISA NA CLÍNICA ODONTOLÓGICA
}

TÍTULO DA PESQUISA: EQUIVALÊNCIA DE INSTRUMENTOS DE QUALIDADE DE VIDA EM SAÚDE BUCAL COM A CLASSIFICAÇÃO INTERNACIONAL DE FUNCIONALIDADE, INCAPACIDADE E SAÚDE

PESQUISADORES: Profa Dra Maria Gabriela Haye Biazevic (orientadora) e Gláucio Umakoshi (mestrando)

ORIENTADOR/PESQUISADOR RESPONSÁVEL: Profa Dra Maria Gabriela Haye Biazevic

OBJETIVO PRINCIPAL DA PESQUISA: Realizar a equivalência de instrumentos de qualidade de vida em saúde bucal (Universidade de Washington - Quality of Life - UW-QoL e Oral Health Impact Profile OHIP com a Classificação Internacional de Funcionalidade, Incapacidade e Saúde (CIF).

Autorizo a utilização da Clínica Odontológica pała a pesquisa acima descrita.

São Paulo, $4,10,2012$

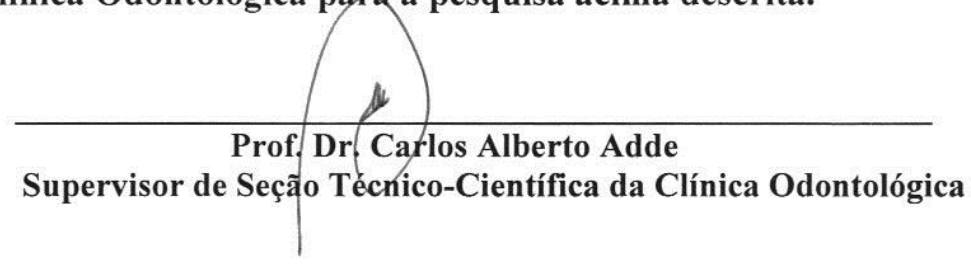

Obs. 1: Caso necessite utilizar prontuários e/ou atendimento a pacientes, a solicitação deverá ser feita oficialmente a Seção de Apoio ao Paciente.

Obs. 2: Lembramos da necessidade da aprovação do Comitê de Ética em Pesquisa para iniciar a pesquisa trazendo cópia do parecer e assinalando os dias de utilização da Clínica Odontológica para a pesquisa.

S.Paulo, 02/10/2012 Nome:MARIA GABRIELA HAYE BIAZEVIC Ass: (nome e assinatura do orientador /pesquisador responsável)

Dia e horário da utilização da clínica para a pesquisa citada acima após aprovação

O mestrando Gláucio Umakoshi frequentará a Clínica Odontológica às $4^{\mathrm{a}}$ feiras, durante o primeiro semestre de 2013. No período da manhã, acompanhará os alunos da Liga de Câncer e aplicará o questionário da Universidade de Washington. No período da tarde, aplicará o questionário OHIP nos pacientes do Setor de Urgência.

Av.Prof.Lineu Prestes, 2227 - Cidade Universitária "Armando de Salles Oliveira" CEP05508-900 São Paulo - SP- Diretoria Telefax: (011) 3091.0062/3091.7817/3091.7860 - Compras (011)3091.7895 
ANEXO B - Termo de Consentimento Livre e Esclarecido Questionário UW-QOL

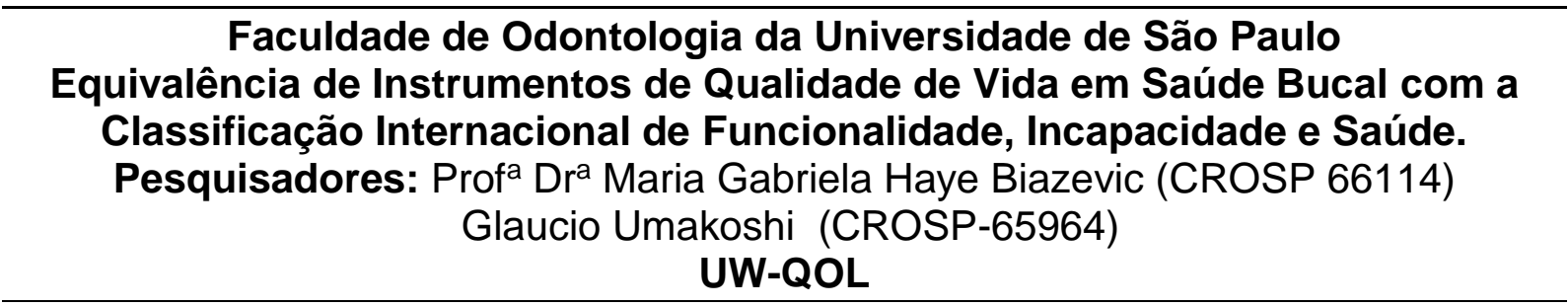

\section{TERMO DE CONSENTIMENTO LIVRE E ESCLARECIDO}

\section{1 - DADOS DE IDENTIFICAÇÃO DO SUJEITO DA PESQUISA}

NOME

SEXO:

M $\square \quad \mathrm{F} \square$ DATA DE NASCIMENTO:

1

DOCUMENTO IDENTIDADE $\mathrm{N}^{\circ}$

ENDEREÇO:

COMPLEMENTO

BAIRRO: CIDADE:

CEP: TELEFONES:

\section{2 - ESCLARECIMENTOS SOBRE A PESQUISA}

O(a) senhor(a) está sendo convidado(a) a participar da pesquisa intitulada "EQUIVALÊNCIA DE INSTRUMENTOS DE QUALIDADE DE VIDA EM SAÚDE BUCAL COM A CLASSIFICAÇÃO INTERNACIONAL DE FUNCIONALIDADE, INCAPACIDADE E SAÚDE". Esta pesquisa tem como objetivo comparar as respostas a questões de qualidade de vida e saúde bucal (University of Washington - Quality of Life (UW-QOL) para os pacientes da Liga de Câncer da Faculdade de Odontologia da Universidade de São Paulo e os códigos da Classificação Internacional de Funcionalidade, Incapacidade e Saúde (CIF). Se o(a) senhor(a) aceitar participar, terá que responder o questionário que faz perguntas a respeito das condições que o senhor(a) percebe em sua boca. Isso deve durar aproximadamente 10 (dez) minutos.

Esta pesquisa está sendo Coordenada pela Prof $^{a}$ Dra $^{\mathrm{a}}$ Maria Gabriela Haye Biazevic (CROSP 66114) e pelo Mestrando Glaucio Umakoshi (CROSP 65964), do Departamento de Odontologia Social da Faculdade de Odontologia da USP. A participação na pesquisa não implicará em nenhuma alteração na rotina do Setor da Liga de Câncer. Sua participação é voluntária e os dados obtidos pela pesquisa serão sigilosos, quer dizer, o seu nome não será divulgado em momento algum. $\mathrm{O}$ (a) senhor(a) poderá retirar o seu consentimento em 
participar da pesquisa a qualquer momento, e não está previsto nenhum benefício direto por participar. Os riscos pela sua participação são mínimos, já que se trata de responder aos questionários.

Não há ajuda de custo. As informações fornecidas serão confidenciais e de conhecimento apenas dos pesquisadores responsáveis. Os participantes da pesquisa não serão identificados em nenhum momento, mesmo quando os resultados desta pesquisa forem divulgados em qualquer forma. Se houver dúvidas sobre a ética da pesquisa entre em contato com o Comitê de Ética em Pesquisa da Faculdade de Odontologia (Av. Lineu Prestes 2227, 05508-000 São Paulo ou pelo e-mail cepfo@usp.br)

\section{3- CONSENTIMENTO LIVRE E ESCLARECIDO}

Declaro ter lido e entendido todas as informações contidas nesse termo, tendo tempo para pensar sobre o assunto e esclarecer minhas dúvidas. Sei que ainda poderei, a qualquer momento, me informar sobre os procedimentos da pesquisa e tirar dúvidas que eventualmente surgirem, pessoalmente ou por telefone, Tel.: (11) 5506-7654 (comercial) ou (11) 9912-5551 (celular). Fui informado(a) que, embora os riscos de participar dessa pesquisa sejam mínimos, na eventualidade de ocorrerem danos a minha saúde, haverá disponibilidade de assistência no Hospital Universitário da USP e na Faculdade de Odontologia da USP.

$\mathrm{Eu}$ portador(a) do RG: concordo em participar voluntariamente desse pesquisa e sei que posso retirar meu consentimento a qualquer momento, sem que isto cause a interrupção da pesquisa a que estou sendo submetido(a) ou qualquer outro tipo de prejuízo. Estou ciente de que os dados obtidos nos meus exames serão mostrados em publicações e eventos científicos sem que eu seja identificado(a). Declaro ter recebido cópia deste termo assinada pelos pesquisadores.

São Paulo, de de 20

Assinatura da participante (sujeito da pesquisa)

Orientadora (Prof ${ }^{a}$ Dra $^{\mathrm{a}}$ Maria Gabriela Haye Biazevic-CROSP:66114)

Orientado (Dr Glaucio Umakoshi-CROSP:65964) 
ANEXO C - Questionário UW-QOL

\begin{tabular}{|c|c|c|}
\hline \multicolumn{2}{|l|}{ Nome: } & Nº Prontuário: \\
\hline \multicolumn{2}{|l|}{ Endereço: } & Bairro: \\
\hline Data Nasc.: ～/ & Tel.: & Sexo: F( ） \\
\hline Motivo da Consulta: & & \\
\hline Diagnóstico: & CID: & \\
\hline \multirow{2}{*}{ Cirurgia: S( ) N( ) } & Quimioterapia S( ) N( ) & Radioterapia S( ) N( ) \\
\hline & Linfonodo: 0()$+(\quad)$ & Metástase S( ) N( ) \\
\hline
\end{tabular}

UW QoL

Este questionário pergunta sobre sua saúde e qualidade de vida durante os últimos sete dias. Por favor, responda a todas as questões marcando uma alternativa para cada questão.

1. Dor (marque uma alternativa $[\mathrm{X}]$ )

100[ ] Eu não tenho dor

75 [ ] Há dor leve não necessitando de medicação

50 [ ] Eu tenho dor moderada, requerendo uso de medicação regularmente

25 [ ] Eu tenho dor severa controlada somente com medicamentos controlados

0 [ ] Eu tenho dor severa, não controlada por medicação

2. Aparência (marque uma alternativa [X])

100 [ ]Não há mudança na minha aparência

75 [ ]A mudança na minha aparência é mínima

50 [ ]Minha aparência me incomoda, mas eu permaneço ativo

25 [ ]Eu me sinto desfigurado significativamente e limito minhas atividades devido a minha aparência

0 [ ]Eu não posso estar com outras pessoas devido a minha aparência

3. Atividade (marque uma alternativa $[X]$ )

100[ ]Eu estou tão ativo quanto sempre estive

75 [ ] Existem vezes em que não posso manter meu ritmo antigo, mas não frequentemente

50 [ ] Eu estou frequentemente cansado e tenho diminuído minhas atividades, embora eu ainda saia de casa

25 [ ]Eu não saio de casa porque eu não tenho força

0 [ ]Eu geralmente fico na cama ou na cadeira e não saio de casa

4. Recreação (marque uma alternativa $[X]$ )

100 [ ]Não há limitações para recreação em casa ou fora de casa

75 [ ] Há poucas coisas que eu não posso fazer, mas eu ainda saio de casa para me divertir

50 [ ]Há muitas vezes em que eu gostaria de sair mais de casa, mas eu não estou bem para isso

25 [ ]Há limitação severa para o que eu posso fazer, geralmente eu fico em casa e assisto à TV

0 [ ] [Eu não posso fazer nada agradável

5. Deglutição (marque uma alternativa $[X]$ )

100 [ ]Eu posso engolir tão bem como sempre

67 [ ]Eu não posso engolir algumas comidas sólidas

33 [ ]Eu posso engolir somente comidas liquidas

0 [ ]Eu não posso engolir porque desce errado e me sufoca 
6. Mastigação (marque uma alternativa $[X]$ )

100[ ]Eu posso mastigar tão bem como sempre

50 [ ]Eu posso comer alimentos sólidos leves mas não consigo mastigar algumas comidas

0 [ ] ]Eu não posso mastigar nem mesmo alimentos leves

7. Fala (marque uma alternativa $[X]$ )

100[ ] Minha fala é a mesma de sempre

67 [ ]Eu tenho dificuldade para dizer algumas palavras mas eu posso ser entendido mesmo ao telefone

33 [ ] Somente minha família e amigos podem me entender

0 [ ] Eu não sou entendido pelos outros

8. Ombro (marque uma alternativa $[\mathrm{X}]$ )

100[ ] Eu não tenho problemas com meu ombro

67 [ ] Meu ombro é endurecido mas isto não afeta minha atividade ou força

33 [ ] Dor ou fraqueza em meu ombro me fizeram mudar meu trabalho

0 [ ] Eu não posso trabalhar devido problemas com meu ombro

9. Paladar (marque uma alternativa [X])

100[ ] Eu sinto sabor da comida normalmente

67 [ ] Eu sinto o sabor da maioria das comidas normalmente

33 [ ] Eu posso sentir o sabor de algumas comidas

0 [ ] Eu não sinto o sabor de nenhuma comida

10. Saliva (marque uma alternativa $[X]$ )

100 [ ]Minha saliva é de consistência normal

67 [ ]Eu tenho menos saliva que o normal, mas ainda é o suficiente

33 [ ]Eu tenho muito pouca saliva

0 [ ]Eu não tenho saliva

11. Humor (marque uma alternativa $[X]$ )

100 [ ]Meu humor é excelente e não foi afetado por causa do meu câncer

75 [ ]Meu humor é geralmente bom e é somente afetado por causa do meu câncer ocasionalmente

50 [ ]Eu não estou nem com bom humor nem deprimido por causa do meu câncer

25 [ ] Eu estou um pouco deprimido por causa do meu câncer

0 [ ]Eu estou extremamente deprimido por causa do meu câncer

12. Ansiedade (marque uma alternativa $[X]$ )

100 [ ]Eu não estou ansioso por causa do meu câncer

67 [ ]Eu estou um pouco ansioso por causa do meu câncer

33 [ ]Eu estou ansioso por causa do meu câncer

0 [ ]Eu estou muito ansioso por causa do meu câncer

13. Quais problemas tem sido os mais importantes para você^ durante os últimos 7 dias? Marque $[X]$ em até 3 alternativas
[ ] Dor
[ ] Deglutição [ ] Paladar
[ ] Aparência [ ] Mastigação [ ] Saliva
[ ] Atividade [ ] Fala [ ] Humor
[ ] Recreação [ ] Ombro [ ] Ansiedade 
14. Questões gerais

Comparado com o mês antes de você desenvolver o câncer, como você classificaria sua qualidade de vida relacionada á saúde.

(marque uma alternativa: [X])

[ ] Muito melhor

[] Um pouco melhor

[ ] Mais ou menos o mesmo

[] Um pouco pior

[] Muito pior

Brief Core Set Questionnaire - Head \& Neck:

Seção 01: Problemas com partes do seu corpo:

1-)Em relação a função da boca em geral?

(0)

(1)

(2)

(3)

(4)

a-) Mordida?

(1)

(2)

(3)

(4)

b-) Mastigação?

(1)

(2)

(3)

(4)

c-) Movendo comida na boca

(0)

d-) Salivação?

(1) (2)

(3)

(4)

(0)

e-) Engolir?

(1)

(2)

(3)

(4)

(0)

(1)

(2)

(3)

f-) Sucção?

(1)

(2)

(3)

(4)

2-) Função da voz em geral?
(0)
(1)
a-) Som que produz?

(2)

(3)

(4)

(2)

(3)

b-) Qualidade do som?

(0)

(1)

(2)

(3)

3-) Função Emocional?

(0)

(1) (2)

(3)

(4)

4-) Energia e condução? (Motivação)

(0)

(1)

(2)

(3)

(4)

5-) Respiração? (Inspirar ou Expirar)
(0)
(1)
(2)
(3)
(4)

6-)Estrutura da boca em geral?

(0) (1) (2)

a-) Estrutura dos dentes

(0) (1) (2)

(3)

(4)

b-) Estrutura dos lábios

(2) (3)

(4)

(0)

(1) (2)

(3)

c-)Estrutura da língua

(0)

(1) (2)

(3)

(4) 

d-) Palato
(0)
(1)
(2)
(3)
(4)
e-) Estrutura de outras partes da boca
(0)
(1)
(2)
(3)
(4)

7-) Estrutura de sua garganta?
(0)
(1)
(2)
(3)
(4)

8-) Estrutura de sua caixa de voz
(0)
(1)
(2)
(3)
(4)?

9-) Estrutura de outras partes de sua cabeça e pescoço?
(0)
(1)
(2)
(3)
(4)

10-) Dor?
(0)
(1)
(2)
(3)
(4)

Opções: (0) nenhum; (1) leve; (2) moderado; (3) grave; (4) completo

Seção 02: Problemas com a atividade social e funcionamento:

1-) Falando?

(0)

(1)

(2)

(3)

(4)

2-) Bebendo?

(0)

(1)

(2)

(3)

(4)

3-) Comendo?

(0)

(2)

(3)

(4)

4-) Realizar sua rotina diária?

(0)

(1)

(2)

(3)

(4)

5-) Apoiando-se financeiramente?
(0)
(1)
(2)
(3)
(4)

6-) Relacionamento familiares?

(0)

(1)

(2)

(3)

(4)

Opções: (0) nenhum; (1) leve; (2) moderado; (3) grave; (4) completo

Seção 03: Problemas com o ambiente:

Quanto sua família tem ajudado ou sido um obstáculo?
$(-4)$
$(-3)$
$(+3)$
$(-2)$
$(-1)$
(0)
$(+1)$
$(+2)$

Quanto aos profissionais de saúde envolvidos no seu atendimento têm sido uma ajuda ou um obstáculo?
$(-4)$
$(-3)$
$(+3)$
$(-2)$
$(+4)$

$(-1)$

(0)

$(+1)$

(+2) 
Quanto os alimentos (sólidos), líquidos, vitaminas etc., que você consome, são um problema ou não?

$(-4)$

$$
\begin{array}{ll}
(-3) & (-2) \\
(+3) & (+4)
\end{array}
$$

$(-1)$

(0)

$(+1)$

Quanto de ajuda ou obstáculo são seus medicamentos (prescritos ou comprados de balcão)?

$(-4)$

$\begin{array}{ll}(-3) & (-2) \\ (+3) & (+4)\end{array}$

$(-1)$

(0)

$(+1)$

(-4) Impedimento Total (+4) Ajuda total 
Faculdade de Odontologia da Universidade de São Paulo Equivalência de Instrumentos de Qualidade de Vida em Saúde Bucal com a Classificação Internacional de Funcionalidade, Incapacidade e Saúde. Pesquisadores: Prof $^{a}$ Dr $^{a}$ Maria Gabriela Haye Biazevic (CROSP 66114) Glaucio Umakoshi (CROSP-65964)

OHIP - 14

TERMO DE CONSENTIMENTO LIVRE E ESCLARECIDO

\section{1 - DADOS DE IDENTIFICAÇÃO DO SUJEITO DA PESQUISA}

NOME

SEXO: $\mathrm{M} \quad \mathrm{F}$ $\square$

DATA DE NASCIMENTO:

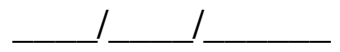

DOCUMENTO

IDENTIDADE $\mathrm{N}^{\circ}$

ENDEREÇO: COMPLEMENTO

BAIRRO: CIDADE:

CEP: TELEFONES:

\section{2 - ESCLARECIMENTOS SOBRE A PESQUISA}

O(a) senhor(a) está sendo convidado(a) a participar da pesquisa intitulada "EQUIVALÊNCIA DE INSTRUMENTOS DE QUALIDADE DE VIDA EM SAÚDE BUCAL COM A CLASSIFICAÇÃO INTERNACIONAL DE FUNCIONALIDADE, INCAPACIDADE E SAÚDE". Esta pesquisa tem como objetivo comparar as respostas à questões de qualidade de vida $\mathrm{e}$ saúde bucal com o questionário da Oral Health Impact Profile (OHIP - 14), para os pacientes da Clínica de Urgências da Faculdade de Odontologia da Universidade de São Paulo e os códigos da Classificação Internacional de Funcionalidade, Incapacidade e Saúde (CIF). Se o(a) senhor(a) aceitar participar, terá que responder este questionário que faz perguntas a respeito das condições que o senhor(a) percebe em sua boca. Isso deve durar aproximadamente 10 (dez) minutos.

Esta pesquisa está sendo Coordenada pela $\operatorname{Prof}^{a} \operatorname{Dr}^{a}$ Maria Gabriela Haye Biazevic (CROSP 66114) e pelo Mestrando Glaucio Umakoshi (CROSP 65964), do Departamento de Odontologia Social da Faculdade de Odontologia da USP. A participação na pesquisa não implicará em nenhuma alteração na rotina do Setor da Clínica de Urgência. Sua participação é voluntária e os dados obtidos pela pesquisa serão sigilosos, quer dizer, o seu nome não será divulgado em momento algum. $\mathrm{O}$ (a) senhor(a) poderá retirar o seu consentimento em 
participar da pesquisa a qualquer momento, e não está previsto nenhum benefício direto por participar. Os riscos pela sua participação são mínimos, já que se trata de responder aos questionários.

Não há ajuda de custo. As informações fornecidas serão confidenciais e de conhecimento apenas dos pesquisadores responsáveis. Os participantes da pesquisa não serão identificados em nenhum momento, mesmo quando os resultados desta pesquisa forem divulgados em qualquer forma. Se houver dúvidas sobre a ética da pesquisa entre em contato com o Comitê de Ética em Pesquisa da Faculdade de Odontologia (Av. Lineu Prestes 2227, 05508-000 São Paulo ou pelo e-mail cepfo@usp.br)

\section{3- CONSENTIMENTO LIVRE E ESCLARECIDO}

Declaro ter lido e entendido todas as informações contidas nesse termo, tendo tempo para pensar sobre o assunto e esclarecer minhas dúvidas. Sei que ainda poderei, a qualquer momento, me informar sobre os procedimentos da pesquisa e tirar dúvidas que eventualmente surgirem, pessoalmente ou por telefone, Tel.: (11) 5506-7654 (comercial) ou (11) 9912-5551 (celular). Fui informado(a) que, embora os riscos de participar dessa pesquisa sejam mínimos, na eventualidade de ocorrerem danos a minha saúde, haverá disponibilidade de assistência no Hospital Universitário da USP e na Faculdade de Odontologia da USP.

$\mathrm{Eu}$ portador(a) do RG: , concordo em participar voluntariamente desse pesquisa e sei que posso retirar meu consentimento a qualquer momento, sem que isto cause a interrupção da pesquisa a que estou sendo submetido(a) ou qualquer outro tipo de prejuízo. Estou ciente de que os dados obtidos nos meus exames serão mostrados em publicações e eventos científicos sem que eu seja identificado(a). Declaro ter recebido cópia deste termo assinada pelos pesquisadores.

São Paulo, de de 20

Assinatura da participante (sujeito da pesquisa)

Orientadora (Prof ${ }^{\mathrm{a}}$ Dr $^{\mathrm{a}}$ Maria Gabriela Haye Biazevic-CROSP:66114)

Orientado (Dr Glaucio Umakoshi-CROSP:65964) 
ANEXO E - Questionário OHIP-14

\begin{tabular}{|l|l|}
\hline Nome: & $N^{\circ}$ Prontuário: \\
\hline Endereço: & Bairro: \\
\hline Data Nasc.: / / Tel.: & Sexo: F ) M ( ) \\
\hline Motivo da Consulta: & \\
\hline Diagnóstico: & \\
\hline
\end{tabular}

OHIP 14

1. Você teve problemas para falar alguma palavra?
(0)
(1)
(2)
(3)
(4)

2. Você sentiu que o sabor dos alimentou tem piorado?
(0)
(1)
(2)
(3)

3. Você sentiu dores em sua boca ou nos seus dentes?
(0)
(1)
(2)
(3)

4. Você se sentiu incomodado(a) ao comer algum alimento?
(0)
(1)
(2)
(3)

5. Você ficou preocupado(a)?
(0)
(1)
(2)

(3)

6. Você se sentiu estressado(a)?

(0) (1)

(2)

(3)

7. Sua alimentação ficou prejudicada?
(0)
(1)
(2)

8. Você teve que
$(0)$
(1)
(2)

(3)
(0)
(1)
(2)
(3)

10. Você se sentiu envergonhado(a)?
(0)
(1)
(2)
(3)

11. Você ficou irritado(a) com outras pessoas?
$(0)$
(1)
(2)
(3)

12. Você teve dificuldade para realizar suas atividades diárias?
(0)
(1)
(2)
(3)

13. Você sentiu que a vida, em geral, ficou pior?
(0)
(1)
(2)
(3)

14. Você ficou total/ incapaz de fazer suas atividades diárias?
(0)
(1)
(2)
(3)

Opções: (0) nunca; (1) raramente; (2) às vezes; (3) repetidamente; (4) sempre 